\title{
A local constitutive model for the discrete element method. Application to geomaterials and concrete
}

\author{
Eugenio Oñate ${ }^{1} \cdot$ Francisco Zárate $^{1} \cdot$ Juan Miquel $^{1} \cdot$ Miquel Santasusana $^{1}$ • \\ Miguel Angel Celigueta ${ }^{1}$ - Ferran Arrufat ${ }^{1}$ - Raju Gandikota ${ }^{2}$. \\ Khaydar Valiullin $^{3}$. Lev Ring ${ }^{3}$
}

Received: 23 January 2015 / Revised: 10 April 2015 / Accepted: 2 May 2015 / Published online: 22 May 2015 (C) OWZ 2015

\begin{abstract}
This paper presents a local constitutive model for modelling the linear and non linear behavior of soft and hard cohesive materials with the discrete element method (DEM). We present the results obtained in the analysis with the DEM of cylindrical samples of cement, concrete and shale rock materials under a uniaxial compressive strength test, different triaxial tests, a uniaxial strain compaction test and
\end{abstract}

Electronic supplementary material The online version of this article (doi:10.1007/s40571-015-0044-9) contains supplementary material, which is available to authorized users.

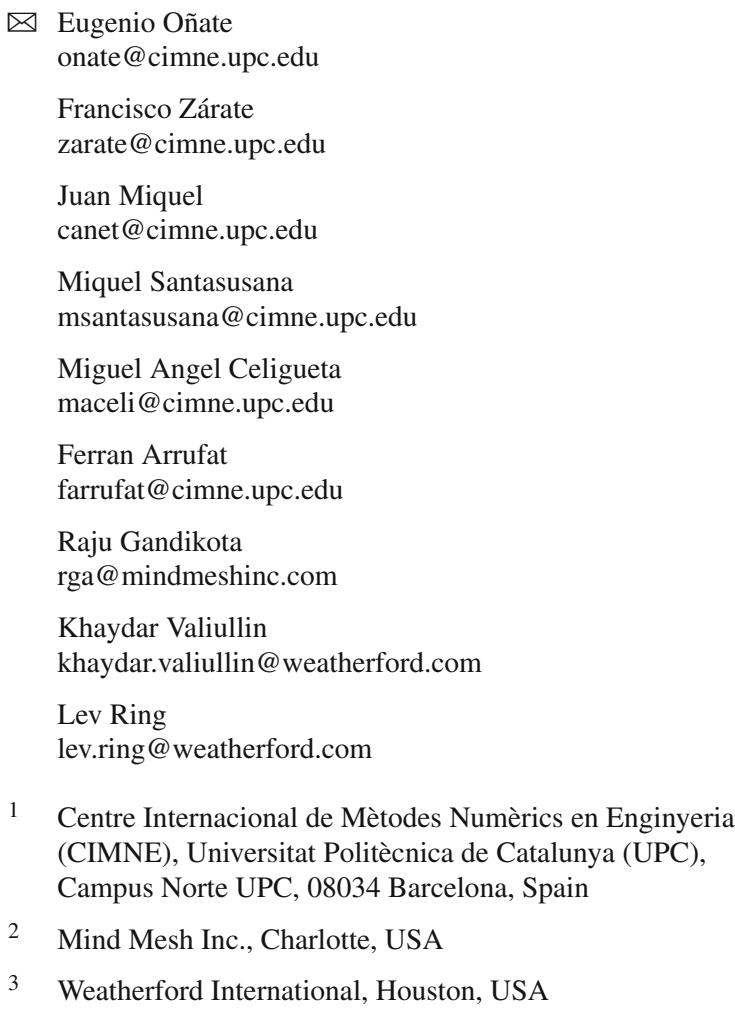

a Brazilian tensile strength test. DEM results compare well with the experimental values in all cases.

Keywords Local constitutive model - Discrete element method · Geomaterials · Concrete

\section{Introduction}

Extensive research work on the discrete element method (DEM) has been carried out in the last decades since the first ideas were presented by Cundall and [11]. Much of the research efforts have focused on the development of adequate DEM models for accurately reproducing the correct behaviour of non cohesive and cohesive granular assemblies $[1,6,8,11,20,24,29,42,45-47]$, as well as of solid materials $[13,14,16-18,22,23,28,31,32,34-36,42,43]$. In recent years the DEM has also been effectively applied to the study of multifracture and failure of geomaterials (soils and rocks), concrete, masonry and ceramic materials, among others.

The analysis of solids with the DEM poses a number of difficulties for adequate reproducing the correct constitutive behaviour of the material under linear (elastic) and non linear conditions.

Within the analysis of solids with the DEM the material is typically represented as a collection of rigid particles [spheres in three dimensions (3D) and discs in two dimensions (2D)] interacting among themselves at the contact interfaces in the normal and tangential directions. Material deformation is assumed to be concentrated at the contact points. Appropriate contact laws are defined in order to obtain the desired macroscopic material properties. The contact law can be seen as the formulation of the material model of the underlying continuum at the microscopic level. For frictional cohesive material the contact law takes into account the cohesive bonds 
between rigid particles. Cohesive bonds can be broken, thus allowing to simulate fracture of the material and its propagation.

A challenge in the failure analysis of solid materials, such as cement, shale rock and concrete, with the DEM is the definition of the limit strengths in the normal and shear directions at the contact interfaces, and the characterization of the non linear relationship between forces and displacements at these interfaces beyond the onset of fracture, accounting for frictional effects, damage and plasticity.

In this work we present a local constitutive model for failure analysis of solid materials typical in geomechanics and concrete applications with the DEM. The model is validated in the analysis of cement, concrete and shale rock samples for several laboratory strength tests. The tests considered include the uniaxial compression strength (UCS) test, triaxial compressive strength tests, the uniaxial strain compaction (USC) test and the Brazilian tensile strength (BTS) test. DEM results compare well with experimental data provided by Weatherford for the cement and shale rock samples $[19,33]$ and the Technical University of Catalonia (UPC) for the concrete samples [37].

\section{Constitutive models for the dem}

Standard constitutive models in the DEM are typically characterized by the following parameters:

Local parameters

- Normal and shear stiffness parameters $K_{n}$ and $K_{s}$.

- Normal and shear strength parameters $\mathcal{F}_{n}$ and $\mathcal{F}_{s}$.

- Coulomb friction coefficient $\mu$.

- Local damping coefficient $C_{n}$.

\section{Global parameters}

- Global damping coefficient for the translational motion, $\alpha^{t}$.

- Global damping coefficient for the rotational motion, $\alpha^{n}$.

Figure 1 shows an scheme of some DEM parameters for a $2 \mathrm{D}$ model.

The challenge in DEM models for analysis of solids is finding an objective and accurate relationship between the DEM parameters and the standard constitutive parameters of a continuum mechanics model (hereafter called "continuum parameters"): the Young modulus $E$, the Poisson ratio $v$ and the tension and shear failure stresses $\sigma_{t}^{f}$ and $\tau^{f}$, respectively.

Two different approaches can be followed for determining the DEM constitutive parameters for a cohesive material, namely the global approach and the local approach. In the global approach uniform global DEM properties are assumed

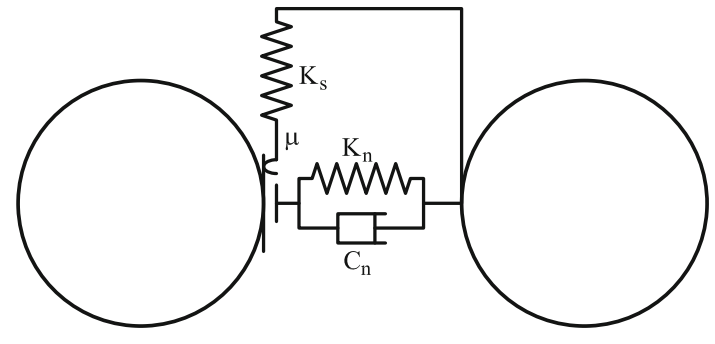

Fig. 1 Model of the contact interface in the DEM

for each contact interface in the whole discrete element assembly. The values of the global DEM parameters can be found via different procedures. Some authors have derived analytical relationships between continuum and global DEM parameters $[24,25]$. Others have used numerical experiments for determining the relationships between DEM and continuum parameters expressed in dimensionless form $[14,17,18]$. This method has been used by the authors in previous works [21-23,31,34-36]. Other procedures are based on relating the global DEM and continuum parameters via laboratory tests using inverse analysis techniques [29].

The local approach used in this work assumes that the DEM parameters depend on the local properties of the interacting particles, namely their radii and the continuum parameters at each interaction point. Different alternatives for defining the DEM parameters via a "local approach" have been reported in recent years [13,14,16,31,32,42,43]. A comparative study of several global and local approaches for estimating the DEM constitutive parameters is presented in [36].

In this work we present a new procedure for defining the DEM parameters for a cohesive material in the framework of the local approach. In the next section we describe how the local elastic parameters can be found. Then we define appropriate local failure criteria at the contact interface using an elasto-damage model for the normal tensile stress and the shear stress, and an elasto-plastic model for the normal compressive stress. The accuracy of the local DEM constitutive model is verified in the analysis of laboratory strength tests for different cohesive materials.

The DEM model presented here can be considered as an extension of that proposed by Donzé and co-workers $[13,16$, $38,43]$. Among the distinct features of our model we note the inclusion of the effect of the size of the interacting spheres in the normal and shear parameters, the introduction of a parameter in the constitutive law accounting for the number of contacts and the packaging of particles, the definition of the failure criteria, the estimation of the limit compressive stress at the contact interface, the effect of damage and plasticity in the evolution of the normal and shear parameters and the definition of the material parameters in terms of the uniaxial stress-strain curve obtained from strength tests. 


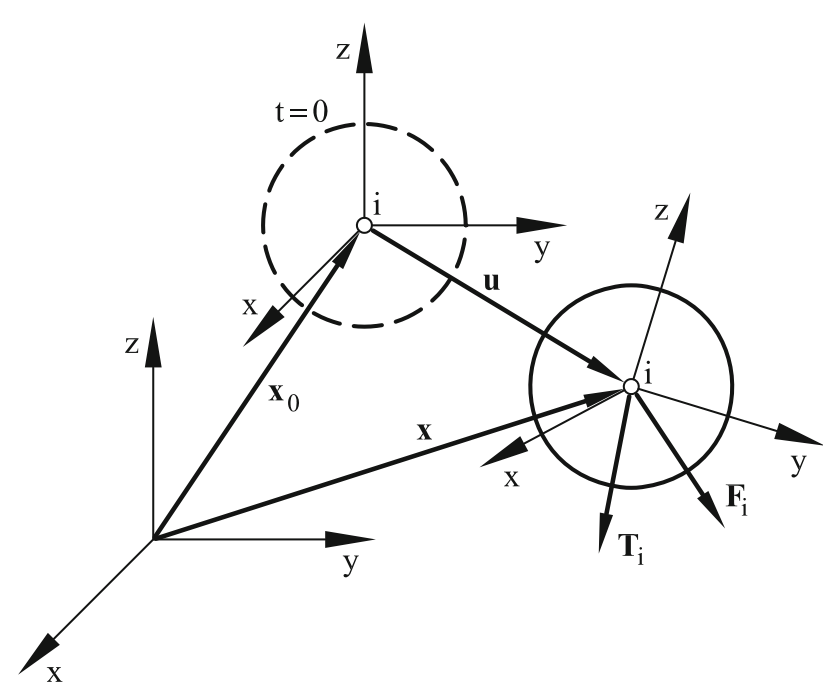

Fig. 2 Motion of a rigid particle

Clearly, the local DEM constitutive model presented in this work is also applicable to standard non-cohesive granular material, as a particular case of the more general expressions for the cohesive case. In this sense, the model is able to simulate the frictional behaviour of the particulate material that forms once the bonds between particles are broken.

\section{Basic equations}

\subsection{Equations of motion}

The translational and rotational motion of rigid spherical or cylindrical particles is described by means of the standard equations of rigid body dynamics. For the $i$-th particle we have (Fig. 2)

$m_{i} \ddot{\mathbf{u}}_{i}=\mathbf{F}_{i}$,

$I_{i} \dot{\omega}_{i}=\mathbf{T}_{i}$,

where $\mathbf{u}_{i}$ is the particle centroid displacement in a fixed (inertial) coordinate frame $\mathbf{X}, \omega_{i}$ - the angular velocity, $m_{i}$ - the particle mass, $I_{i}$ - the moment of inertia, $\mathbf{F}_{i}$-the resultant force, and $\mathbf{T}_{i}$ - the resultant moment about the central axes. Vectors $\mathbf{F}_{i}$ and $\mathbf{T}_{i}$ are sums of: (i) all forces and moments applied to the $i$-th particle due to external loads, $\mathbf{F}_{i}^{e x t}$ and $\mathbf{T}_{i}^{e x t}$, respectively, (ii) contact interactions with neighbouring particles $\mathbf{F}^{i j}$ (Fig. 3), $j=1, \ldots, n_{i}^{c}$, where $n_{i}^{c}$ is the number of particles being in contact with the $i$-th particle, (iii) forces and moments resulting from external damping, $\mathbf{F}_{i}^{d a m p}$ and $\mathbf{T}_{i}^{d a m p}$, respectively. Thus, we can be write as

$$
\mathbf{F}_{i}=\mathbf{F}_{i}^{e x t}+\sum_{j=1}^{n_{i}^{c}} \mathbf{F}^{i j}+\mathbf{F}_{i}^{\text {damp }}
$$
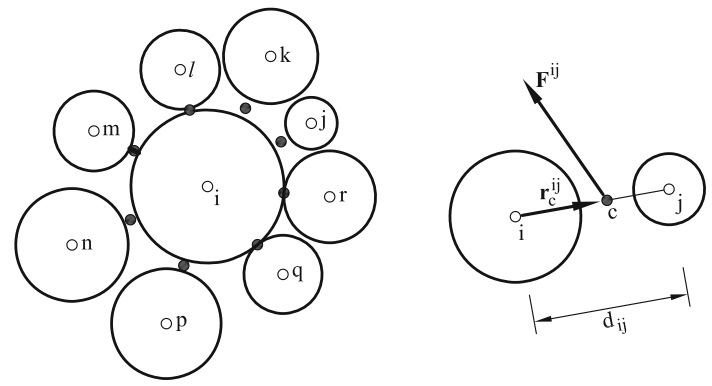

Fig. 3 Force $\mathbf{F}^{i j}$ at the contact interface between particles $i$ and $j$

$\mathbf{T}_{i}=\mathbf{T}_{i}^{e x t}+\sum_{j=1}^{n_{i}^{c}} \mathbf{r}_{c}^{i j} \mathbf{F}^{i j}+\mathbf{T}_{i}^{\text {damp }}$

where $\mathbf{r}_{i j}^{c}$ is the vector connecting the centroid of the $i$-th particle with the contact point $c$ at the interface between particles $i$ and $j$ (Fig. 3).

The form of the rotational motion of Eq. (2) holds for spheres and cylinders (in 2D) and is simplified with respect to a general form for an arbitrary rigid body with the rotational inertial properties represented by a second order tensor. In the general case it is more convenient to describe the rotational motion with respect to a co-rotational frame $\mathbf{x}$ which is embedded at each element, since in this frame the tensor of inertia is constant.

\subsection{Integration of the equations of motion}

Equations (1) and (2) are integrated in time using a standard central difference scheme $[31,48]$. The time integration operator for the translational motion at the $n$-th time step is as follows:

$\ddot{\mathbf{u}}_{i}^{n}=\frac{\mathbf{F}_{i}^{n}}{m_{i}}$,

$\dot{\mathbf{u}}_{i}^{n+1 / 2}=\dot{\mathbf{u}}_{i}^{n-1 / 2}+\ddot{\mathbf{u}}_{i}^{n} \Delta t$

$\mathbf{u}_{i}^{n+1}=\mathbf{u}_{i}^{n}+\Delta u_{i} \quad$ with $\quad \Delta u_{i}=\dot{\mathbf{u}}_{i}^{n+1 / 2} \Delta t$

The first two steps in the integration scheme for the rotational motion are identical to those given by Eqs. (5) and (6):

$\dot{\omega}_{i}^{n}=\frac{\mathbf{T}_{i}^{n}}{I_{i}}$,

$\omega_{i}^{n+1 / 2}=\omega_{i}^{n-1 / 2}+\dot{\omega}_{i}^{n} \Delta t$

The vector of incremental rotation $\Delta \boldsymbol{\theta}=\left[\Delta \theta_{x}, \Delta \theta_{y}\right.$, $\left.\Delta \theta_{z}\right]^{\mathrm{T}}$ is calculated for the $i$ th particle as

$\Delta \boldsymbol{\theta}_{i}=\omega_{i}^{n+1 / 2} \Delta t$ 
Knowledge of the incremental rotation suffices to update the tangential contact forces. It is also possible to track the rotational position of particles, if necessary. Then the rotation matrices between the moving frames embedded in the particles and the fixed global frame must be updated incrementally using an adequate multiplicative scheme [23,31,48].

Explicit integration in time yields high computational efficiency and it enables the solution of large models. The disadvantage of the explicit integration scheme is its conditional numerical stability imposing the limitation on the time step $\Delta t[48]$, i.e.

$\Delta t \leq \Delta t_{\mathrm{cr}}$

where $\Delta t_{\mathrm{cr}}$ is a critical time step determined by the highest natural frequency of the system $\omega_{\max }$ as

$\Delta t_{\mathrm{cr}}=\frac{2}{\omega_{\max }}$

If damping exists, the critical time increment is given by

$\Delta t_{\mathrm{cr}}=\frac{2}{\omega_{\max }}\left(\sqrt{1+\xi^{2}}-\xi\right)$

where $\xi$ is the fraction of the critical damping corresponding to the highest frequency $\omega_{\max }$. Exact calculation of the highest frequency $\omega_{\max }$ requires the solution of the eigenvalue problem defined for the whole system of connected rigid particles. In an approximate solution procedure, an eigenvalue problem can be defined separately for every rigid particle using the linearized equations of motion

$\mathbf{m}_{i} \ddot{\mathbf{a}}_{i}+\mathbf{k}_{i} \mathbf{a}_{i}=\mathbf{0}$

where

$\mathbf{m}_{i}=\left\{m_{i}, m_{i}, m_{i}, I_{i}, I_{i}, I_{i}\right\}^{\mathrm{T}}$,

$\mathbf{a}_{i}=\left\{\left(u_{x}\right)_{i},\left(u_{y}\right)_{i},\left(u_{z}\right)_{i},\left(\theta_{x}\right)_{i},\left(\theta_{y}\right)_{i},\left(\theta_{z}\right)_{i}\right\}^{\mathrm{T}}$

and $\mathbf{k}_{i}$ is the stiffness matrix accounting for the contributions from all the interface constraints that are active for the $i$-th particle.

\section{Frictional contact conditions}

\subsection{Contact interface}

Let us assume that an individual particle is connected to the adjacent ones by appropriate relationships at the contact interfaces. These relationships define either a perfectly bond or a frictional sliding situation at the interface.

Particles are assumed to be spherical and can have very different sizes. Each particle $i$ is characterized by the sphere radius $r_{i}$. We will assume that particles $i$ and $j$ are in contact at a point $c$ located at a distance $(1+\beta) r_{i}$ or $(1+\beta) r_{j}$ from the centers of particles $i$ and $j$, respectively (Fig. 4) where $\beta$ is a positive number. Typically, $0 \leq \beta \leq 0.20$. We define

Fig. 4 Definition of contact interface. $\mathbf{a} \beta \neq 0$. $\mathbf{b} \beta=0$

(a) Contact interface $(\beta \neq 0)$
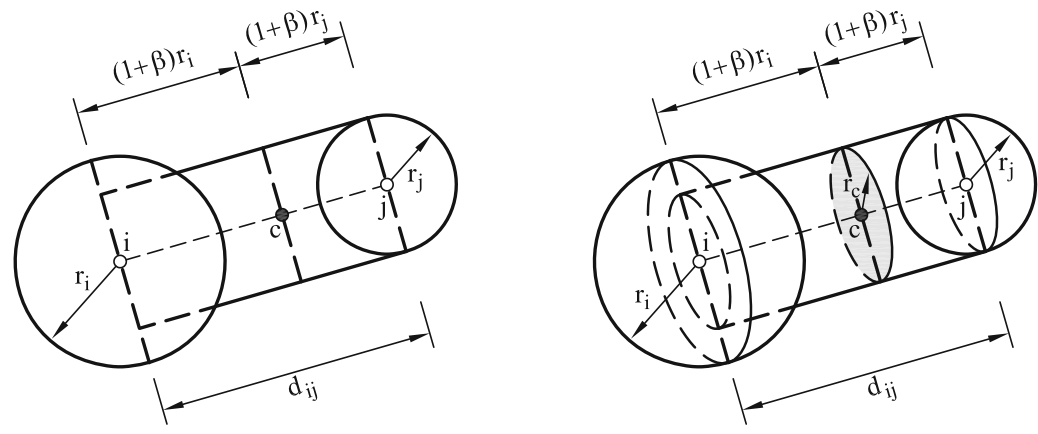

(b) Contact interface $(\beta=0)$
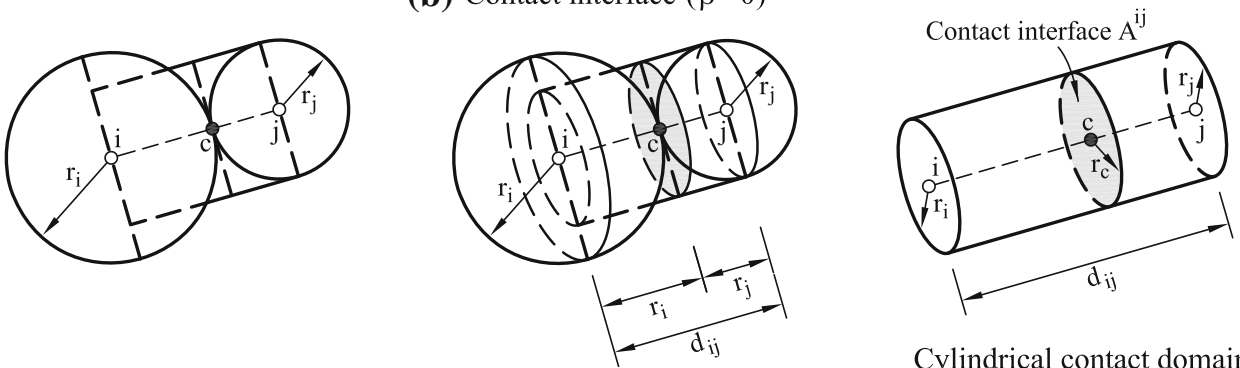

Cylindrical contact domain 
the interaction domain between the two particles that share the contact point $c$ as a cylinder of radius equal to the radius of the smaller of the two particles in contact (Fig. 4). The circular section at point $c$ of radius $r_{c}$ is the contact interface between particles $i$ and $j$.

This definition of the contact interface and the interaction domain is motivated by the fact that the two interacting particles can have very different radius for an arbitrary distribution of the particle sizes. The contact interface is thus limited by the size of the smaller of the two particles in contact.

\subsection{Interaction range}

The overall mechanical behaviour of a material can be reproduced by associating a simple constitutive law to each contact interface. The interaction between spherical (in 3D) or circular (2D) particles $i$ and $j$ with radius $r_{i}$ and $r_{j}$, respectively is defined within an interaction range. This range allows for a certain gap or an overlapping between the particles. Then two particles will interact if

$1-\beta \leq \frac{d_{i j}}{r_{i}+r_{j}} \leq 1+\beta$

where $d_{i j}$ is the distance between the centroids of particles $i$ and $j$ and $\beta$ is the interaction range parameter (Figs. 3, 4). This choice is made so that the DEM can simulate materials other than simple granular materials, in particular those which involve a matrix, as it is found for instance in concrete, cement and rock. A value of $\beta>0$ is chosen in order to model the effect of this matrix that may glue two aggregates which are not themselves in contact. In this manner the model can handle the presence of gaps and overlappings generated by most of the sphere meshers. Simply, the contact search is extended using a value of $\beta>0$ and the equilibrium position of the contact point is then set for its initial configuration (which is not always a tangential contact of particles). This works inwards for particle inclusions or outwards for gaps. For the problems solved in this work we have taken $\beta=0.10$.

From Eq. (16) we deduce

$d_{i j}=(1 \pm \beta)\left(r_{i}+r_{j}\right)$

where the plus and minus sign accounts for the gap and overlapping between the two particles, respectively.

\subsection{Contact search algorithm}

Changing contact pairs of elements during the analysis are automatically detected. The simple approach to identify interaction pairs by checking every particle against every other one would be very inefficient, as the computational time
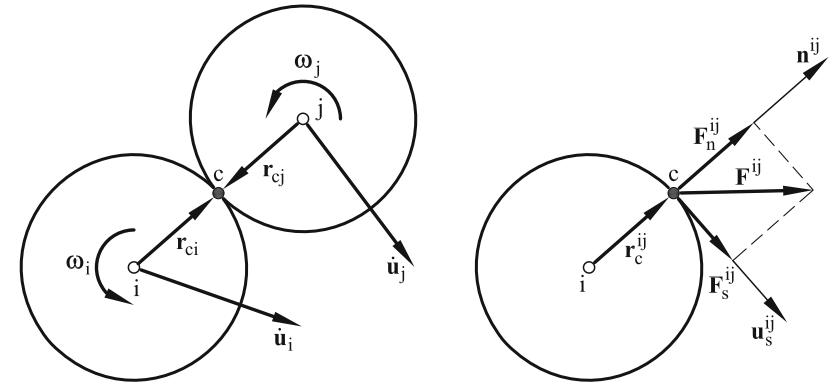

Fig. 5 Decomposition of the contact force into its normal and tangential components

is proportional to $n^{2}$, where $n$ is the number of elements. In our formulation the search is performed using a grid-based algorithm. In this case the computation time of the contact search is proportional to $n \ln n$, which allows us to solve large frictional contact systems involving many particles [23].

\subsection{Decomposition of the contact force}

Once contact between a pair of particles has been detected, the forces occurring at the contact point are calculated. The interaction between the two interacting particles can be represented by the contact forces $\mathbf{F}^{i j}$ and $\mathbf{F}^{j i}$, which satisfy the following relation:

$\mathbf{F}^{i j}=-\mathbf{F}^{j i}$

We decompose $\mathbf{F}^{i j}$ into its normal and shear components, $\mathbf{F}_{n}^{i j}$ and $\mathbf{F}_{s}^{i j}$, respectively (Fig. 5)

$\mathbf{F}^{i j}=\mathbf{F}_{n}^{i j}+\mathbf{F}_{s}^{i j}=F_{n} \mathbf{n}^{i j}+\mathbf{F}_{s}^{i j}$

where $\mathbf{n}^{i j}$ is the unit vector normal to the contact interface between particles $i$ and $j$ and $F_{n}$ is the modulus of the normal force at the interface. Equation (18b) implies that the normal force lies along the line connecting the centers of the two particles in contact and directed outwards from particle $i$ (Fig. 5).

The shear force $\mathbf{F}_{s}^{i j}$ along the shear direction (Fig. 6) can be written as

$\mathbf{F}_{s}^{i j}=F_{s_{1}} \mathbf{s}_{1}+F_{s_{2}} \mathbf{s}_{2}$

where $F_{S_{1}}$ and $F_{S_{2}}$ are the shear force components along the shear directions $s_{1}$ and $s_{2}$, and $\mathbf{s}_{1}$ and $\mathbf{s}_{2}$ are unit vectors in these directions. Vector $\mathbf{s}_{1}$ is taken in an arbitrary direction orthogonal to the normal vector. Then $\mathbf{s}_{2}=\mathbf{n}^{i j} \times \mathbf{s}_{1}$.

The shear force modulus $F_{S}$ is obtained as

$F_{s}^{i j}=\left|\mathbf{F}_{s}^{i j}\right|=\left(F_{s_{1}}^{2}+F_{s_{2}}^{2}\right)^{1 / 2}$ 


\section{Interface section $A^{\mathrm{ij}}$}

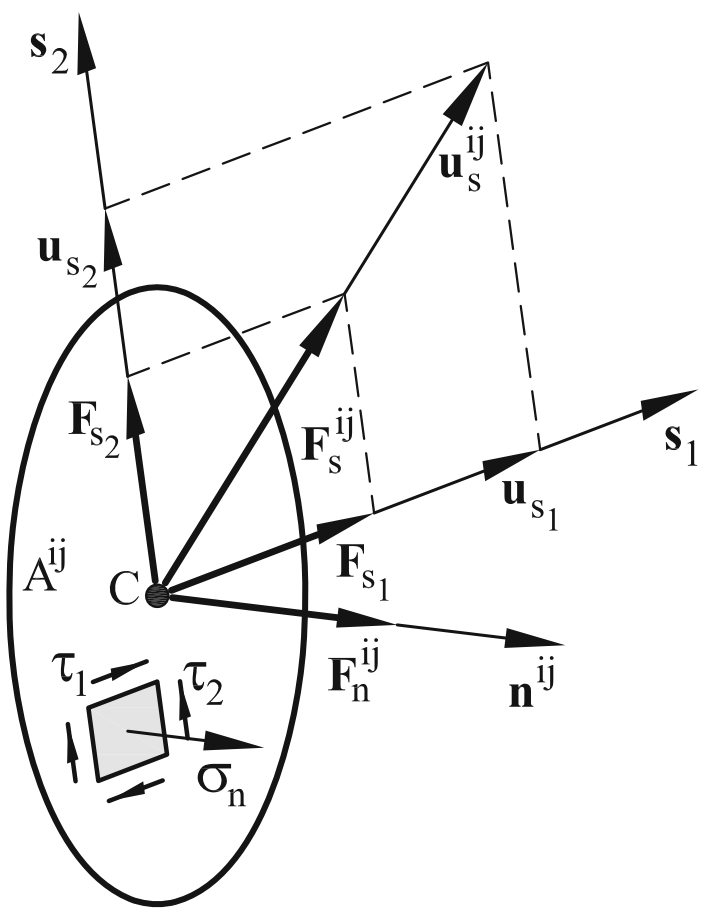

Fig. 6 Forces and stresses acting on a contact interface section $A^{i j}$. Definition of normal and shear directions

The relationship between the contact forces $F_{n}, F_{S_{1}}$ and $F_{S_{2}}$ and the particle displacements are obtained using the local constitutive model described in the next section.

\subsection{Definition of the contact law}

In this work we have assumed a proportionality between the normal force at each contact interfaces and the relative displacement and the relative velocity of the contact point. As for the shear force, this has been assumed to be proportional to the relative sliding motion at the contact point. The proportionality coefficients have been estimated starting from the one-dimensional stress-strain relationship for the cylindrical contact domain of Fig. 4. This approach has been preferred versus the contact laws proposed by Hertz [15] and Mindlin [27] for modelling the contact interaction between two spheres in the normal and tangential directions, respectively. These laws have been used to model the contact force in granular material with the DEM $[4,46]$. For the solids material considered in this work, the existence of a matrix between grains [(modelled via a gap distance, Eq. (16)] prevents in most cases the direct contact between particles, which justifies the more "diffusive" contact model chosen in this work.
A comparison of different contact has shown that simple models in the DEM contact models, such as that presented here, lead to equivalent and, sometimes even better, results than more sophisticated models [12].

\section{Local definition of dem elastic constitutive parameters}

\subsection{Normal force parameters}

The normal force $F_{n}$ at the contact interface between particles $i$ and $j$ is obtained as

$F_{n}^{i j}=\sigma_{n} \bar{A}^{i j}$

where $\sigma_{n}$ is the normal stress at the contact interface and $\bar{A}^{i j}$ is the effective area at the interface computed as

$\bar{A}^{i j}=\alpha_{i j} A^{i j}$ with $A^{i j}=\pi r_{c}^{2}$

Recall that $r_{c}$ is the radius of the smaller of the two particles interacting at the interface $i j$ (Fig. 4).

In Eq. (21) $\alpha_{i j}$ is a parameter that accounts for the fact that the number of contacts and the packaging of particles are not optimal. Clearly, $\alpha_{i j}$ is a local parameter for the $i j$ interface. In this work, however, we have considered the case of spherical particles only and used a global definition of $\alpha_{i j}$ as

$\alpha_{i j}=\alpha=40 \frac{P}{N_{c}}$

where $N_{c}$ and $P$ are the average number of contacts per sphere and the average porosity for the whole particle assembly. Equation (22) has been deduced by defining the optimal values for the number of contacts per sphere and for the global porosity equal to $10 \%$ and $25 \%$, respectively. Clearly $\alpha \simeq 1$ for quasi-optimal packaging distributions, as it is the case for the particle meshes using in the examples solved in this work

The normal stress $\sigma_{n}$ is related to the normal strain between the interacting spheres, $\varepsilon_{n}$, by a visco-elastic law as

$\sigma_{n}=E \varepsilon_{n}+c \dot{\varepsilon}_{n}$

where $E$ is the Young modulus of the solid material and $c$ is a damping coefficient.

We note that the Young modulus is assumed to be an intrinsic property of the material. As such it is typically characterized from axial tests on cylindrical samples. Thus, the Young modulus obtained from the experimental axial 
stress-strain relationship is used for defining the local normal stress-strain relationship at a macroscopic level (23) at the contact interfaces. The same applies to the definition of the Poisson's ratio of the material used for defining the local shear constitutive relationship in Sect. 5.2.

The normal strain and the normal strain rate are defined as

$\varepsilon_{n}=\frac{u_{n}}{d_{i j}}, \quad \dot{\varepsilon}_{n}=\frac{\dot{u}_{n}}{d_{i j}}$

where $d_{i j}$ is given by Eq. (17).

Substituting Eq. (24) into (23) gives

$\sigma_{n}=\frac{1}{d_{i j}}\left[E u_{n}+c \dot{u}_{n}\right]$

In Eqs. (24) and (25) $u_{n}$ and $\dot{u}_{n}$ are the normal (relative) displacement and the normal (relative) velocity at the contact point defined as

$u_{n}=\left(\mathbf{x}_{j}-\mathbf{x}_{i}\right) \cdot \mathbf{n}^{i j}-d_{i j}, \quad \dot{u}_{n}=\left(\dot{\mathbf{u}}_{j}-\dot{\mathbf{u}}_{i}\right) \cdot \mathbf{n}^{i j}$

where $\mathbf{x}_{i}$ and $\mathbf{x}_{j}$ are the position vectors of the centroids of particles $i$ and $j$. Note that $u_{n}$ and $\dot{u}_{n}$ express the relative (incremental) motion between the centroids of the interacting particles.

The damping coefficient $c$ is taken as a fraction $\xi$ of the critical damping $\bar{c}$ per unit length for the system of two rigid spherical bodies with masses $m_{i}$ and $m_{j}$ connected by a spring of stiffness $K_{n}[23,31]$, i.e.

$c=\frac{\xi \bar{c}}{r_{c}}=2 \frac{\xi}{r_{c}} \sqrt{m_{i j} K_{n}}$

with $0<\xi \leq 1$ and $m_{i j}$ is the reduced mass of the contact,

$m_{i j}=\frac{m_{i} m_{j}}{m_{i}+m_{j}}$

In our work we have taken $\xi=0.9$.

From Eqs. (20) and (25) we deduce the relationship between the normal force and the normal relative motion at the interface between particles $i$ and $j$ as

$F_{n}^{i j}=\frac{\bar{A}^{i j}}{d_{i j}}\left(E u_{n}+c \dot{u}_{n}\right)=K_{n} u_{n}+C_{n} \dot{u}_{n}$

Substituting Eqs. (21) and (27) into (29) we find the expression of the normal stiffness and the normal viscous (damping) coefficients at the contact interface as

$K_{n}=\frac{\alpha_{i j} \pi r_{c}^{2}}{d_{i j}} E \quad C_{n}=\frac{2 \pi r_{c} \alpha_{i j} \xi}{d_{i j}} \sqrt{m_{i j} K_{n}}$
Equation (29) is assumed to hold in the elastic regime for both the normal tensile force $F_{n_{t}}$ and the normal compressive force $F_{n_{c}}$.

\subsection{Shear force parameters}

A similar approach is followed for obtaining the relationship between the shear forces and the relative tangential displacements at each contact interface.

The shear forces in the $s_{1}$ and $s_{2}$ directions (Fig. 6) are given by

$F_{s_{1}}=\tau_{1} \bar{A}^{i j}, \quad F_{S_{2}}=\tau_{2} \bar{A}^{i j}$

where $\tau_{1}$ and $\tau_{2}$ are the shear stresses at the contact interface. These stresses are linearly related to the shear strains $\gamma_{1}$ and $\gamma_{2}$ at the interface by

$\tau_{1}=G \gamma_{1}, \quad \tau_{2}=G \gamma_{2}$

where $G$ is the shear modulus.

A simple definition of the shear strains is

$\gamma_{1}=\frac{u_{s_{1}}}{d_{i j}}, \quad \gamma_{2}=\frac{u_{s_{2}}}{d_{i j}}$

where $u_{s_{1}}$ and $u_{s_{2}}$ are the components in the $s_{1}$ and $s_{2}$ directions of the relative tangential displacement vector at the contact point given by (Fig. 6)

$\mathbf{u}_{s}^{i j}=\left[u_{s_{1}}, u_{s_{2}}\right]^{T}=\mathbf{u}^{i j}-\left(\mathbf{u}^{i j} \cdot \mathbf{n}^{i j}\right) \mathbf{n}^{i j}$

with

$\mathbf{u}^{i j}=\left(\Delta \mathbf{u}_{i}+\left(\omega_{i} \times \mathbf{r}_{c_{i}}\right) \Delta t\right)-\left(\Delta \mathbf{u}_{j}+\left(\boldsymbol{\omega}_{j} \times \mathbf{r}_{c_{j}}\right) \Delta t\right)$

where $\Delta \mathbf{u}_{i}$ and $\Delta \mathbf{u}_{j}$ are the displacement increments of the centroids of particles $i$ and $j$, respectively [Eq. (7)] and $\mathbf{r}_{c_{i}}$ and $\mathbf{r}_{c_{j}}$ are the vectors connecting the particle centers with the contact point (Fig. 5).

Substituting Eqs. (32) and (33) into (31) we find

$F_{s_{1}}=K_{s_{1}} u_{s_{1}} \quad$ and $\quad F_{s_{2}}=K_{s_{2}} u_{s_{2}}$

with

$K_{s_{1}}=K_{s_{2}}=K_{s}=\frac{K_{n}}{2(1+v)}$

where $v$ is the Poisson ratio.

From Eq. (35) we can obtain a relationship between the modulus of the shear force and the modulus of the shear displacement vector as

$F_{s}=K_{s} u_{s}$ 
with

$$
\begin{aligned}
& F_{S}=\left|\mathbf{F}_{s}^{i j}\right|=\left[\left(F_{S_{1}}\right)^{2}+\left(F_{S_{2}}\right)^{2}\right]^{1 / 2}, \\
& u_{s}=\left|\mathbf{u}_{s}^{i j}\right|=\left[\left(u_{s_{1}}\right)^{2}+\left(u_{s_{2}}\right)^{2}\right]^{1 / 2}
\end{aligned}
$$

The sign of $F_{s_{k}}(k=1,2)$ in Eq. (35) depends on the sign of the velocity component $u_{s_{k}}$, while in Eq. (37) only the modulus of vectors $\mathbf{F}_{s}^{i j}$ and $\mathbf{u}_{s}^{i j}$ are involved.

For convenience the upper indices $i, j$ are omitted hereonwards in the expression of the normal and shear force vectors $\mathbf{F}_{n}^{i j}$ and $\mathbf{F}_{s}^{i j}$ at a contact interface.

\section{Global background damping force}

A quasi-static state of equilibrium for the assembly of particles can be achieved by application of an adequate global damping to all particles. This damping adds to the local one introduced at the contact interface (Sect. 5.1). We have considered the following non-viscous type global damping terms

$$
\begin{aligned}
\mathbf{F}_{i}^{\text {damp }} & =-\alpha^{t}\left|\mathbf{F}_{i}^{\text {ext }}+\mathbf{F}^{i j}\right| \frac{\dot{\mathbf{u}}_{i}}{\left|\dot{\mathbf{u}}_{i}\right|} \\
\mathbf{T}_{i}^{\text {damp }} & =-\alpha^{r}\left|\mathbf{T}_{i}\right| \frac{\boldsymbol{\omega}_{i}}{\left|\boldsymbol{\omega}_{i}\right|}
\end{aligned}
$$

The definition of the translational and rotational damping parameters $\alpha^{t}$ and $\alpha^{r}$ is a topic of research. A practical alternative is to define $\alpha^{t}$ and $\alpha^{r}$ as a fraction of the stiffness parameters $K_{n}$ and $K_{S}$, respectively. In our work we have taken $\alpha^{t}=\alpha^{r}=0.1$. Alternative a viscous type damping can be used, as described in $[23,31]$.

\section{Elasto-damage model for tension and shear forces}

\subsection{Normal and shear failure}

In this model, cohesive bonds at a contact interface are assumed to start breaking when the interface strength is exceeded in the normal direction by the tensile contact force, or in the tangential direction by the shear force. The uncoupled failure (decohesion) criterion for the normal and tangential directions at the contact interface between particles $i$ and $j$ is written as

$F_{n_{t}} \geq \mathcal{F}_{n_{t}}, \quad F_{s} \geq \mathcal{F}_{s}$

where $\mathcal{F}_{n_{t}}$ and $\mathcal{F}_{s}$ are the interface strengths for pure tension and shear-compression conditions, respectively, $F_{n_{t}}$ is the normal tensile force and $F_{S}$ is the modulus of the shear force vector defined in Eq. (38).
The interface strengths are defined as

$\mathcal{F}_{n_{t}}=\sigma_{t}^{f} \bar{A}^{i j}, \quad \mathcal{F}_{s}=\tau^{f} \bar{A}^{i j}+\mu_{1}\left|F_{n_{c}}\right|$

where $\sigma_{t}^{f}$ and $\tau^{f}$ are the tensile and shear failure stresses, respectively (also called tensile and shear strengths), $F_{n_{c}}$ is the compressive normal force at the contact interface and $\mu_{1}=\tan \phi_{1}$ is a (static) friction parameter, where $\phi_{1}$ is an internal friction angle. These values are assumed to be an intrinsic property of the material and are determined experimentally. In our work $\sigma_{t}^{f}$ is taken as the tensile strength of the material measured in a bending-tensile (BT) test (i.e. $\left.\sigma_{t}^{f}=\left(\sigma_{t}^{f}\right)_{B T}\right)$. The value of $\sigma_{t}^{f}$ can also be obtained from the failure stresses in a Brazilian tensile strength (BTS) test using the relationship between the tensile strength in BTS and BT tests. For the examples presented in this paper we have accepted that $\sigma_{f}^{t}=\left(\sigma_{t}^{f}\right)_{B T}=1.60\left(\sigma_{t}^{f}\right)_{B T S}[2,5]$.

In absence of values from specific tensile strength tests the tensile strength $\sigma_{t}^{f}$ can also be estimated from the maximum compressive stress, $\left(\sigma_{n_{c}}^{f}\right)_{\mathrm{UCS}}$, in a uniaxial compressive strength (UCS) test in a cylindrical sample. A typical relationship for concrete, used in this work, is

$\sigma_{t}^{f}=0.464\left[\left(\sigma_{n_{c}}^{f}\right)_{U C S}\right]^{2 / 3}$

There is a big discrepancy in the literature for the value of the shear strength $\tau^{f}$ for frictional cohesive materials. In our work we have estimated the value of $\tau^{f}$ as a percentage of the maximum compressive stress in an UCS test, $\left(\sigma_{n_{c}}^{f}\right)_{U C S}$, as

$\tau^{f}=\beta\left(\sigma_{n_{c}}^{f}\right)_{U C S}$

where $\beta$ is a parameter that is calibrated in numerical experiments via UCS and BTS tests. In our numerical experiments for cement, concrete and shale rock materials the value of $\beta$ used has ranged from $0.4<\beta<0.55$. The corresponding value of $\tau^{f}$ is in agreement with the experimental results of Talbot [41] and Anderson et al. [3] for estimating the shear strength of cement and concrete materials more than are century ago, as well as with more recent experimental data for concrete and rocks $[9,10,39,40,44]$.

Following tension failure, the constitutive behavior in the shear direction is governed by the standard Coulomb law

$\mathbf{F}_{s}=\mu_{2}\left|F_{n_{c}}\right| \frac{\mathbf{u}_{s}}{\left|\mathbf{u}_{s}\right|} \quad$ with $\quad \mu_{2}=\tan \phi_{2}$

where $\mu_{2}$ is a dynamic Coulomb friction coefficient and $\phi_{2}$ is the post-failure internal friction angle. Both $\mu_{2}$ and $\phi_{2}$ are determined from experimental tests.

Figure 7a shows the graphical representation of the failure criterium described by Eqs. (41), (42) and (45). This criterium 
Fig. 7 Failure line in terms of normal and shear forces. a Uncoupled failure model. b Coupled failure model

Fig. 8 Undamaged and damaged elastic moduli under tension (a) and shear (b) forces

(a)

(b)

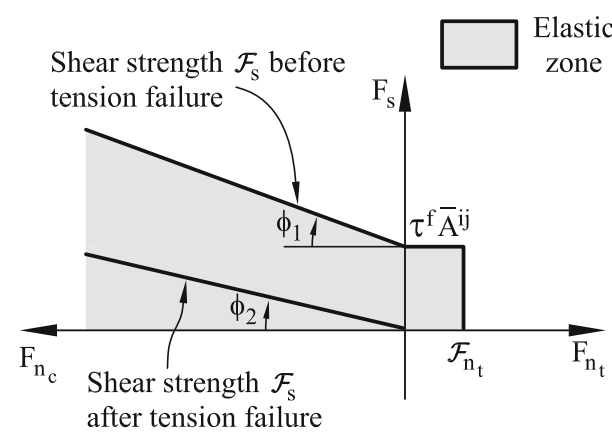

(a) Failure under tension

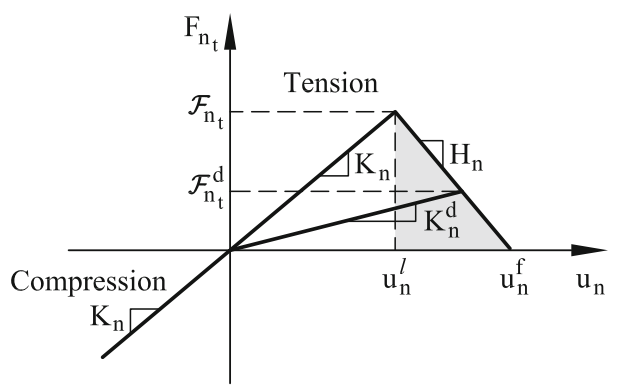

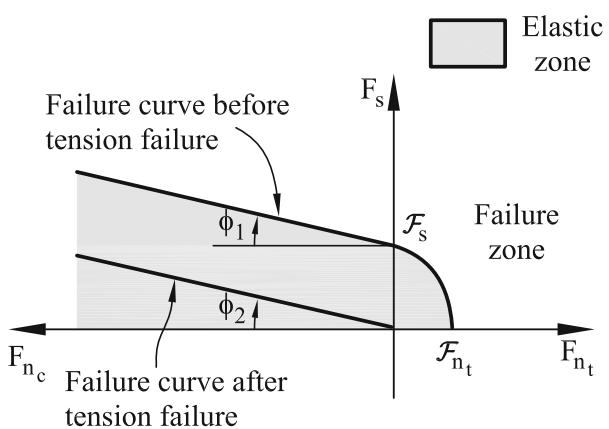

(b) Failure under shear

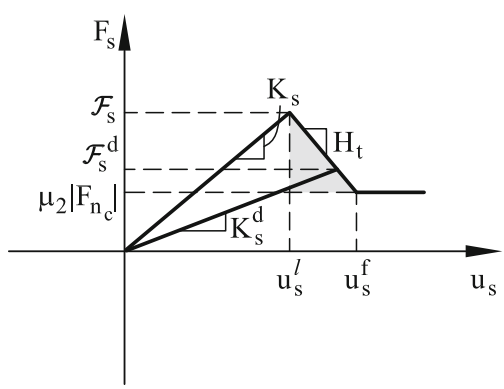

assumes that the tension and shear forces contribute to the failure of the contact interface in a decoupled manner. On the other hand, shear failure under normal compressive forces follows a failure line that is a function of the shear failure stress, the compression force and the internal friction angle.

Indeed, a coupled failure model in the tension-shear zone can also be used, as shown in Fig. 7b. For the numerical tests presented in the paper the uncoupled model has been used.

Figure 8 shows the evolution of the normal tension force $F_{n_{t}}$ and the shear force $F_{S}$ at a contact interface until failure in terms of the relative normal and tangential displacements. The effect of damage in the two constitutive laws is also shown in the figure. The method for introducing damage in the constitutive equations is explained in the next section.

\subsection{Damage evolution law}

Elastic damage under tensile and shear conditions has been taken into account in this work by assuming a linear softening behaviour defined by the softening moduli $H_{n}$ and $H_{t}$ introduced into the force-displacement relationships in the normal (tensile) and shear directions, respectively (Fig. 8).

The constitutive relationships for the elasto-damage model are written as

\section{Normal (tensile) direction}

For $0<d_{n} \leq 1: F_{n_{t}}=\left(1-d_{n}\right) K_{n} \frac{u_{n}^{l}}{u_{n}} u_{n}=K_{n}^{d} u_{n}$

with $\quad K_{n}^{d}=\left(1-d_{n}\right) \frac{u_{n}^{l}}{u_{n}} K_{n}$
For $d_{n} \geq 1: F_{n_{t}}=0$

\section{Shear direction}

For $0<d_{s} \leq 1: F_{s}=\left(1-d_{s}\right) K_{s} \frac{u_{s}^{l}}{u_{s}} u_{s}=K_{s}^{d} u_{s}$

with $\quad K_{s}^{d}=\left(1-d_{s}\right) \frac{u_{s}^{l}}{u_{s}} K_{s}$

For $d_{s}>1: F_{s}=\mu_{2}\left|F_{n_{c}}\right|$

where $d_{n}$ and $d_{s}$ are scalar damage parameters in the normal (tensile) and shear directions at the contact interface, respectively and $K_{n}^{d}$ and $K_{s}^{d}$ are damaged elastic stiffness parameters. The damage parameters $d_{n}$ and $d_{s}$ are a measure of the loss of mechanical strength at each contact interface. For the undamaged state $d_{n}=0$ and $d_{s}=0$, while for a damaged state $0<d_{n} \leq 1$ and $0<d_{s} \leq 1$.

Damage effects are assumed to start when the strength failure conditions (41) are satisfied. The evolution of the damage parameters from the value zero to one can be defined in a number of ways using fracture mechanics arguments. A key issue is that the area under the line defining the force (relative) displacement relationship in the damaged region (the shadowed area in Fig. 8) equals the specific fracture energy of the material $[26,30,31]$.

The following damage parameters are defined for convenience 
$\delta_{n}=\frac{u_{n}^{f}-u_{n}^{l}}{u_{n}^{l}}, \quad \delta_{s}=\frac{u_{s}^{f}-u_{s}^{l}}{u_{s}^{l}}$

where $u_{n}^{f}$ and $u_{s}^{f}$ are values of the interface displacement increments in the normal and shear directions at failure computed as

$u_{n}^{f}=d_{i j} \varepsilon_{n}^{f}, \quad u_{s}^{f}=\sqrt{\bar{A}^{i j}} \gamma_{s}^{f}$

where $\varepsilon_{n}^{f}$ and $\gamma_{s}^{f}$ are respectively the failure normal strain and the failure shear strain. These strains are an intrinsic property of the material that has to be obtained experimentally.

In our work we have defined the damage parameters $d_{n}$ and $d_{s}$ in Eq. (46) using a simple linear strain softening law as

$d_{n}= \begin{cases}0 & \text { for } u_{n}<u_{n}^{l}=\frac{\mathcal{F}_{n_{t}}}{K_{n}} \\ \frac{1}{\delta_{n}}\left(\frac{u_{n}}{u_{n}^{l}}-1\right) & \text { for } u_{n}^{l} \leq u_{n}<u_{n}^{f} \\ 1 & \text { for } u_{n} \geq u_{n}^{f}\end{cases}$
$d_{s}= \begin{cases}0 & \text { for } u_{s}<u_{s}^{l}=\frac{\mathcal{F}_{s}}{K_{s}} \\ \frac{1}{\delta_{s}}\left(\frac{u_{s}}{u_{s}^{l}}-1\right) & \text { for } u_{s}^{l} \leq u_{s}<u_{s}^{f} \\ 1 & \text { for } u_{s} \geq u_{s}^{f}\end{cases}$

The failure conditions evolve due to damage as follows

$F_{n_{t}} \geq \mathcal{F}_{n_{t}}^{d}, \quad F_{s} \geq \mathcal{F}_{s}^{d}$

with

$\mathcal{F}_{n_{t}}^{d}=\mathcal{F}_{n_{t}}-H_{n}\left(u_{n}-u_{n}^{l}\right)$

$\mathcal{F}_{s}^{d}=\mathcal{F}_{s}-H_{t}\left(u_{s}-u_{s}^{l}\right)$

where $\left(\mathcal{F}_{n_{t}}, \mathcal{F}_{n_{t}}^{d}\right)$ and $\left(\mathcal{F}_{s}, \mathcal{F}_{s}^{d}\right)$ are the undamaged and damage interface strengths for pure tension and pure shear conditions, respectively.

Figure 9 shows the evolution of the failure lines from the undamaged to the fully damaged state for the uncoupled model of Fig. 7a.

\section{Elasto-plastic model for compression forces}

The compressive stress-strain behaviour in the normal direction at the contact interface for frictional cohesive materials, such as cement, rock and concrete, is typically governed by an initial elastic law followed by a non-linear constitutive

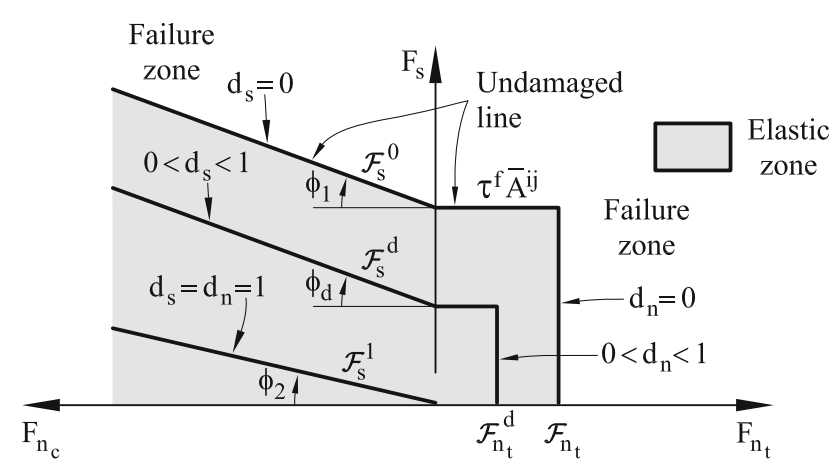

Fig. 9 Evolution of the failure lines due to damage for uncoupled normal and shear failure

equation that varies for each material. The compressive normal stress increases under linear elastic conditions until it reaches the limit normal compressive stress $\sigma_{n_{c}}^{l}$ (also called yield stress). This is defined as the axial stress level where the experimental curve relating the axial stress and the axial strain starts to deviate from the linear elastic behaviour. After this point the material is assumed to yield under elasticplastic conditions.

The elasto-plastic relationships in the normal compressive direction are defined as

\section{Loading path}

$d F_{n_{c}}=K_{T_{n}} d u_{n}$

Unloading path

$d F_{n_{c}}=K_{n_{0}} d u_{n}$

In Eq. (52) $d F_{n_{c}}$ and $d u_{n}$ are respectively the increment of the normal compressive force and the normal (relative) displacement, $K_{n_{0}}$ is the initial (elastic) compressive stiffness for a value of $E=E_{0}$ (Fig. 10), and $K_{T_{n}}$ is the tangent compressive stiffness given by

$K_{T_{n}}=\frac{E_{T}}{E_{0}} K_{n_{0}}$

where $E_{T}$ is the slope of the normal stress-strain curve in the elastoplastic branch (i.e. $K_{T}=E_{1}, E_{2}, E_{3}$ in Fig. 10).

Plasticity effects in the normal compressive direction also affect the evolution of the tangential forces at the interface, as the interface shear strength is related to the normal compression force by Eq. (42).

Figure 10 shows the diagram relating the compressive axial stress and the compressive axial strain used for modelling the elasto-plastic constitutive behaviour at the contact interfaces. The form of each diagram is typically obtained from experimental tests on cylindrical samples with the adjustment explained in the next section. 


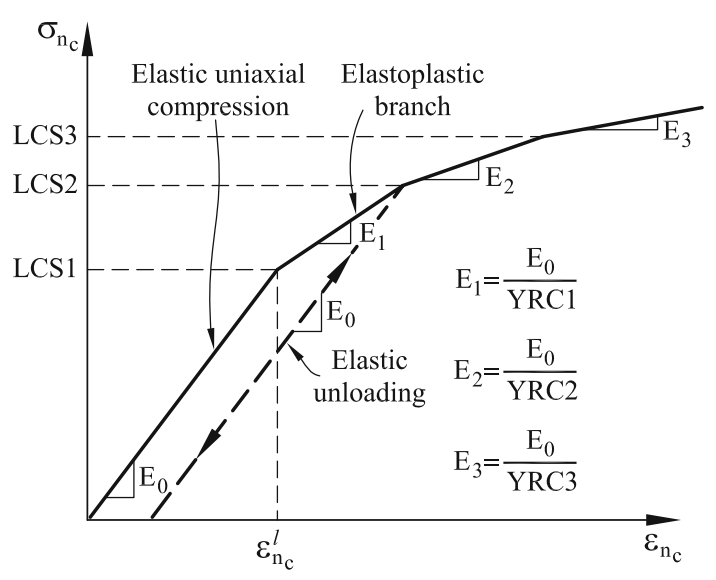

Fig. 10 Compressive axial stress-compressive axial strain diagram for elastoplastic material. LCS1 $=\sigma_{n_{c}}^{l}$ : limit compressive stress dening the onset of elastoplastic behaviour at the contact interface

The diagram in Fig. 10 will be used for modelling with the DEM the tests in cement, concrete and shale rock material in this work.

\section{Limit compressive stress at the contact interface: experimental adjustment}

The limit compressive stress at the contact interface is obtained by correcting the experimental value of the limit compressive stress obtained in a UCS test. The correction is needed for taking into account the micro-macro relationship that relates the limit normal stress at the contact interfaces with the limit compressive stress obtained from experimental tests.

Figure 11 shows the average values of the ratio $r=\frac{\sigma_{n_{c}}^{l}}{\sigma_{a}^{l}}$ at all the contact interfaces in terms of the angle that the normal vector to the interface forms with the horizontal axis for different triaxial tests on cement samples. In the expression of $r, \sigma_{n_{c}}^{l}$ is the limit normal compressive stress at the contact interface and $\sigma_{a}^{l}$ is the limit compressive stress obtained in a UCS test. The results displayed in Fig. 11 shows that the values of $r$ range form $0.55-0.65$ for UCS and triaxial tests.

The conclusion of this study is that the limit normal compressive stress at the contact interface, $\sigma_{n_{c}}^{l}$, is a proportion of the actual limit compressive stress in a experimental test. In our work we have computed $\sigma_{n_{c}}^{l}$ as $\sigma_{n_{c}}^{l}=0.62\left(\sigma_{n_{c}}^{l}\right)_{U C S}$, where $\left(\sigma_{n_{c}}^{l}\right)_{U C S}$ is the yield stress obtained in a UCS test.

\section{Numerical experiments}

The DEM model presented in the previous sections has been implemented in the DEMPACK code (www.cimne. com/dempack), based on routines from the open-source object-oriented software platform KRATOS (www.cimne.

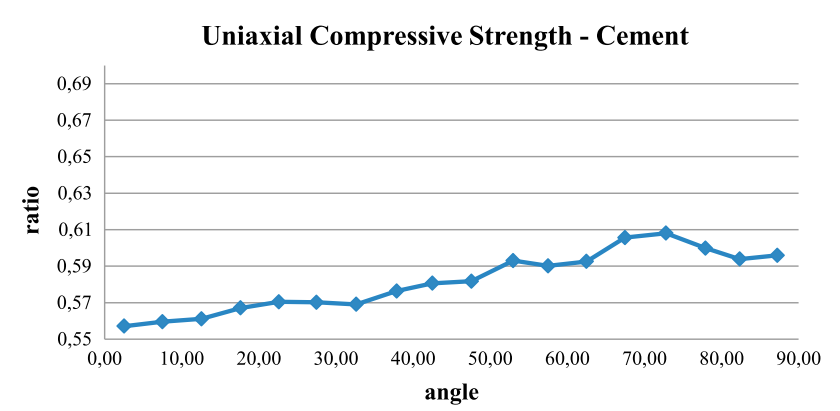

Triaxial 500Psi - Cement

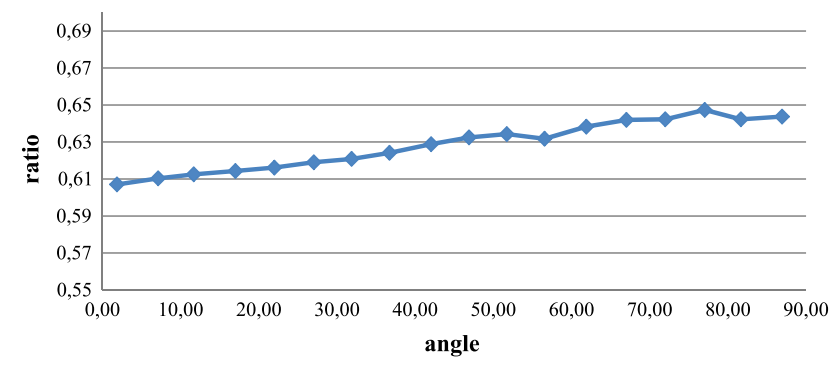

Triaxial 1000Psi - Cement

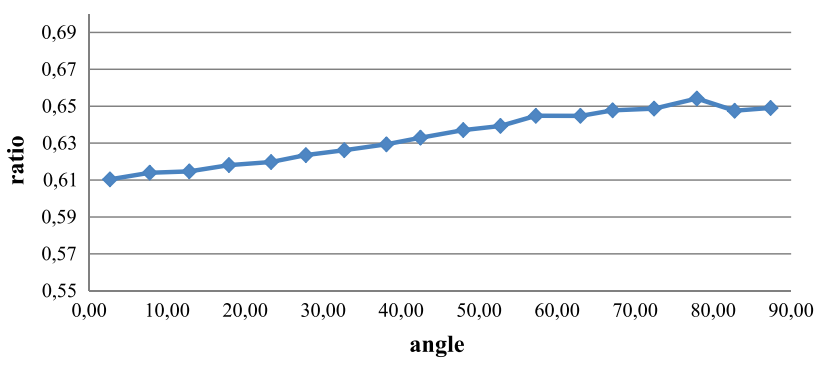

Triaxial 2000Psi - Cement

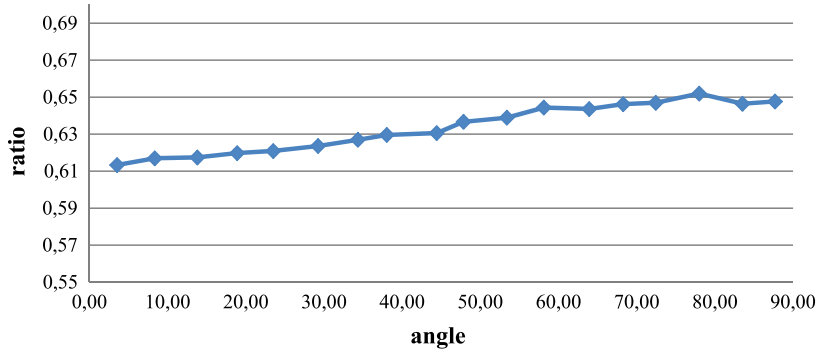

Fig. 11 Micro-macro failure compressive stress ratios $\left(r=\frac{\sigma_{n_{c}}^{l}}{\sigma_{a}^{l}}\right)$ obtained for cement samples

com/kratos) and the pre-postprocessing system GiD (www. gidhome.com). DEMPACK is a fully parallelized DEM code. Table 1 shows the computer times and speed-ups for the analysis of a UCS test with a DEM mesh of 70,000 spheres and 1000 time steps using different processors in a Intel Xeon ES-2670 machine $(2 \times 8=$ cores $)$ using OpenMP and MPI parallel computing strategies. Typically, the MPI strategy provided a better speed up in all cases. Note that the problem was solved in some $56 \mathrm{~s}$ using 16 processors. Indeed, this 
Table 1 CPU times (in seconds) and speed-ups for parallel analysis of UCS test (70,000 spheres) using MPI and OpenMP strategies

\begin{tabular}{lcccc}
\hline $\begin{array}{l}\text { No. of } \\
\text { processors }\end{array}$ & $\begin{array}{l}\text { OpenMP } \\
\text { (time, s) }\end{array}$ & $\begin{array}{l}\text { OpenMP } \\
\text { (speed-up) }\end{array}$ & $\begin{array}{l}\text { MPI } \\
\text { (time, s) }\end{array}$ & $\begin{array}{l}\text { MPI } \\
\text { (speed-up) }\end{array}$ \\
\hline 1 & 699,25 & 1 & 699,25 & 1 \\
2 & 434,03 & 1,735 & 372,29 & 1,878 \\
4 & 255,35 & 3,083 & 243,92 & 2,867 \\
8 & 151,23 & 5,301 & 111,01 & 6,299 \\
12 & 111,05 & 7,175 & 71,09 & 9,725 \\
16 & 88,04 & 8,563 & 56,35 & 12,410 \\
\hline
\end{tabular}

speed can be improved by enhancing the parallel computing features of the code.

\subsection{DEM analysis of laboratory tests on cement samples}

\subsubsection{UCS and triaxial tests on cement samples}

The experimental procedure for the triaxial tests on cement samples studied in this work is as follows:

(1) A right cylindrical plug is cut from the sample core and its ends ground parallel each other within 0.001 inch. Physical dimensions and weight of the specimen are recorded. The dimensions of the cylindrical samples are 1 inch diameter and 2 inch height. The specimen is tested under saturated condition with water.

(2) The specimen is then placed between two endcaps and a heat-shrink jacket is placed over the specimen.

(3) Axial strain and radial strain devices are mounted in the endcaps and on the lateral surface of the specimen, respectively.

(4) The specimen assembly is placed into the pressure vessel and the pressure vessel is filled with hydraulic oil.

(5) Confining pressure is increased to the desired hydrostatic testing pressure.

(6) Specimen assembly is brought into the contact with a loading piston that allows application of axial load.

(7) Increase axial load at a constant rate until the specimen fails or axial strain reaches a desired amount of strain while confining pressure is held constant.

(8) Reduce axial stress to the initial hydrostatic condition after sample fails or reaches a desired axial strain.

(9) Reduce confining pressure to zero and disassemble sample.

The simulation of a triaxial test with the DEM reproduces the experiment as follows.

(a) The confining pressure is applied up to the desired hydrostatic testing pressure. (b) A prescribed axial motion is applied at the top of the specimen until this fail, or the axial compressive strain strain reaches a desired amount of strain while confining pressure is held constant.

For the UCS test the process starts by step (b) above described with zero confinement pressure.

We note that the goal of this study was to reproduce with the DEM model presented the structural behaviour of the sample during the axial compression phase. For this purpose an average Young modulus (deduced from the uniaxial strain compaction (USC) test) was chosen for modelling the the hydrostatic compaction of the sample during the application of the confining pressure. A more detailed study of the non linear behaviour of the cement sample under an hydrostatic load will be presented in a subsequent work.

Figure 12 shows the normal stress-strain relationship for a cement material as deduced from the USC test presented in [19]. The curve shows an initial elastic branch and a (elastoplastic) hardening branch.

Figure 13 shows the so-called "differential stress" computed as the difference between the applied axial stress and the confinement pressure during the USC test for the same cement material [19]. The curve shows the initial linear elastic part, a limit axial stress of around $10 \mathrm{Mpa}$ and the subsequent non linear branch. The non linear region has a flat part which indicates the compaction of the cement material for that stress level. This is followed by a hardening branch which evidences the recovery of the material stiffness for high compaction values.

For completeness, Figure 14 shows the evolution of confinement pressure during the USC test. We note that the curve in Figure 13 is the difference between the curves of Figures 12 and 14.

The stress in the curve in Figure 13 coincides with the effective stress only if we accept that the water pressure in the pores is the same as the external pressure required to enforce the uniaxial strain conditions during the test.

In our work we have used the stress-strain curve obtained in the USC test as the basis for computing the normal compressive force at the contact interfaces $\left(\sigma_{a}\right)$ for the cement material examples. Note that for saturated conditions this curve already accounts for the effect of water pressure at the pores.

Tables 2, 3 and 4 show the material and DEM parameters for the cement material studied in this work. Table 2 shows the basic material parameters reported in [19]. The tensile strength $\sigma_{t}^{f}$ has been deduced from the BTS test value of $\left(\sigma_{t}^{f}\right)_{B T S}=2.92 \mathrm{MPa}$ [19] using the relationship $\sigma_{t}^{f}=$ $1.60\left(\sigma_{t}^{f}\right)_{B T S} \simeq 4.80 \mathrm{MPa}$ as mentioned in Sect. 7.1. On the other hand, $\tau^{f}$ has been taken as $\tau^{f}=\frac{1}{2}\left(\sigma_{n_{c}}^{f}\right)_{U C S}=8.50$ $\mathrm{MPa}$, where $\left(\sigma_{n_{c}}^{f}\right)_{U C S}$ is the maximum compressive stress obtained in the UCS test. 
Fig. 12 Uniaxial strain compaction test in cement sample [19]. Normal total compressive stress-axial strain relationship

Fig. 13 Uniaxial strain compaction test in cement sample [19]. Differential stress between the applied axial stress and the confinement pressure
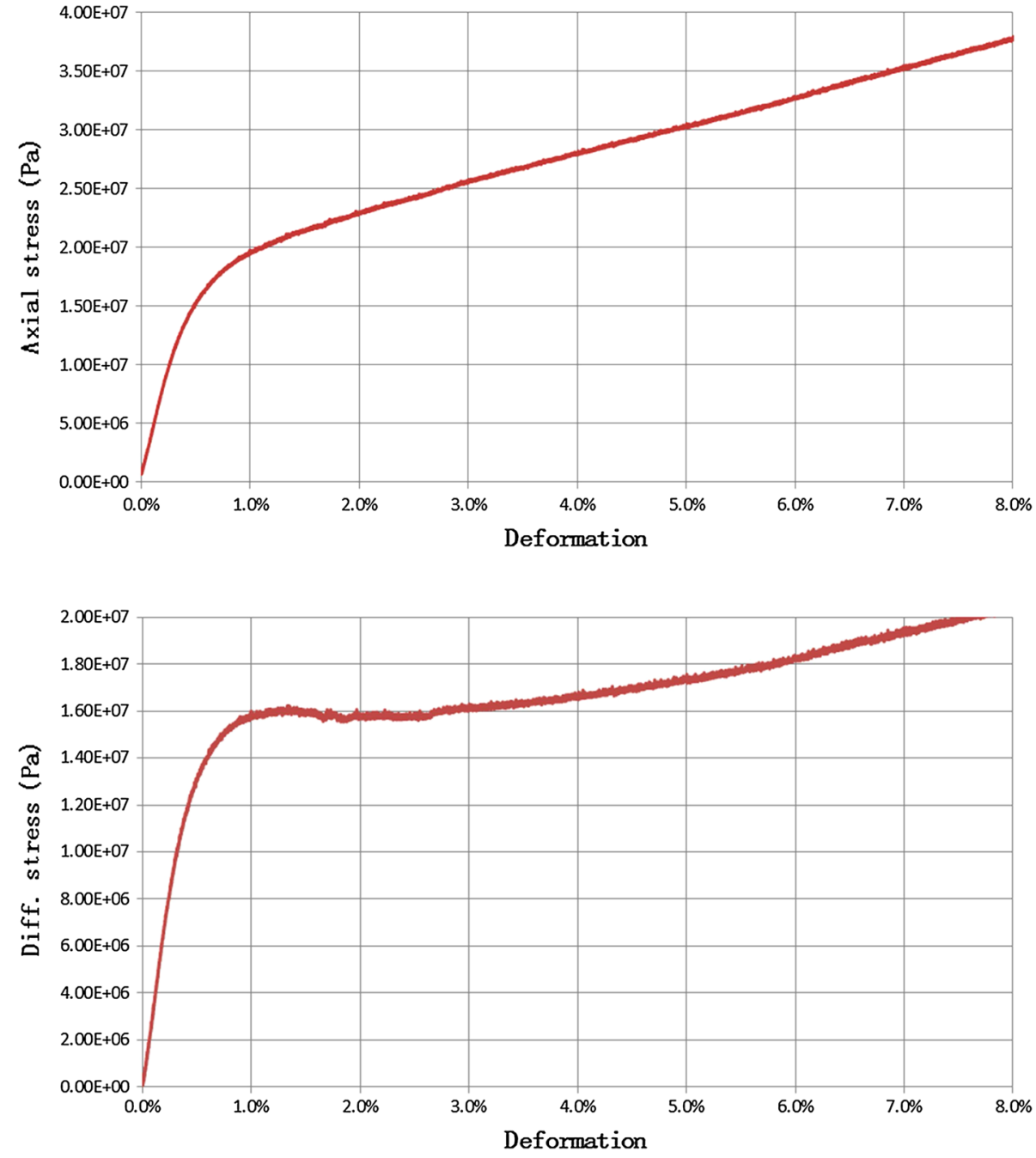

Fig. 14 Uniaxial strain compaction test in cement sample [19]. Confinement pressure versus axial deformation

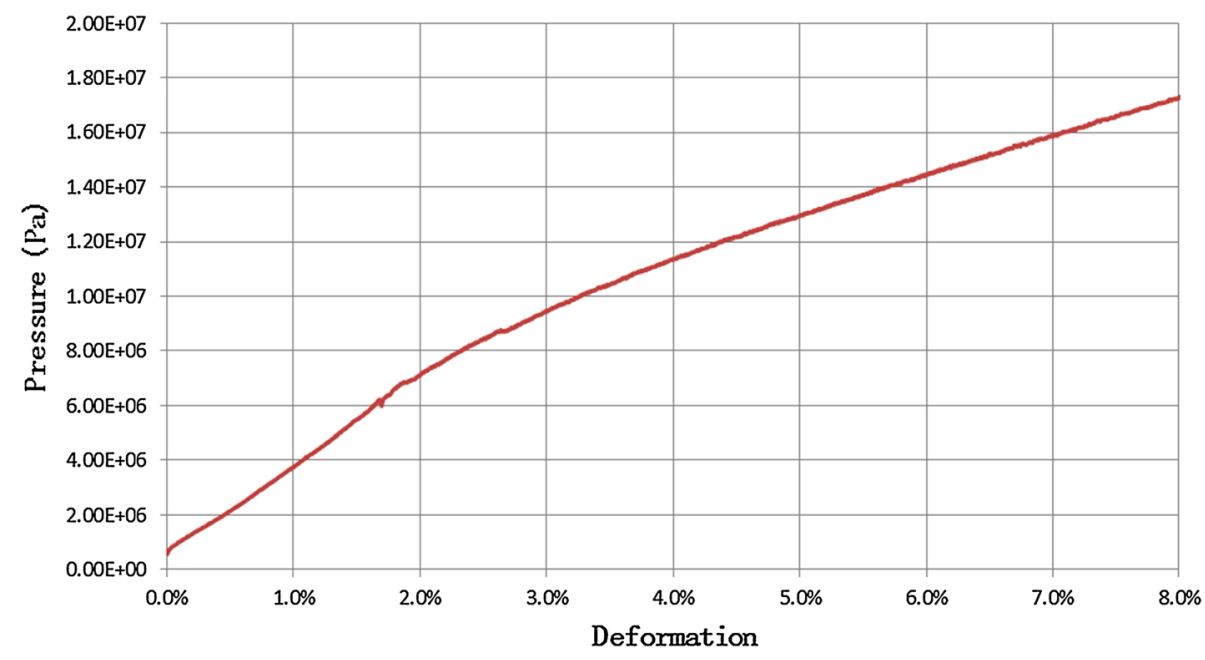

Table 3 shows the DEM constitutive parameters for the UCS, USC and BTS tests corresponding to the values defined in Figure 10. Table 4 lists the DEM constitutive parameters for triaxial tests.
The confining pressure is directly applied to the spheres that lay on the surface of the specimen. A normal force is applied to each surface particle in the radial direction. The magnitude of the force has been approximated in this work 
Table 2 Material parameters for cement

\begin{tabular}{lllllll}
\hline$\rho(\mathrm{g} / \mathrm{cc})$ & $\mu_{1}$ & $\mu_{2}$ & $E_{0}(\mathrm{GPa})$ & $v$ & $\sigma_{t}^{f}(\mathrm{MPa})$ & $\tau^{f}(\mathrm{MPa})$ \\
\hline 1.70 & 0.30 & 0.40 & 3.80 & 0.20 & 4.80 & 8.50 \\
\hline
\end{tabular}

as $F_{n_{i}}=\pi r_{i}^{2} p_{c}$ where $r_{i}$ is the particle radius and $p_{c}$ is the confining pressure. A more accurate procedure for transferring the confining pressure to the spheres at the boundary of the specimen using the areas of the Voronoi polygon created by the centroids of the spheres is presented in [7].

A small confining pressure of 70 Psi was applied for the analysis of the UCS test. This pressure reproduces the effect of the heat-shrink jacket on the lateral deformation of the sample.

Figures 15 and 16 show DEM results for the UCS and triaxial tests for a discretization of 42000 spheres.

Figure 15 shows DEM results of the applied axial versus the axial strain in the cement specimen for the UCS test. Figure 16 shows DEM results for triaxial tests in the cement samples for confining pressures of 500, 1000, 2000 and 4000 Psi using again 42000 spheres. Good correlation between the DEM results and the experimental values [19] is obtained in all cases.
Figure 17 shows the deformation of the sample at the failure point for the UCS test where the typical cracking pattern can be seen. A similar set of results is shown in Fig. 18 for the triaxial test under a confining pressure of 500 Psi and different deformation levels.

\subsubsection{Uniaxial strain compaction (USC) test on cement sample}

For the USC test the radial strain is constrained in the sample while a piston presses the sample from the top. The DEM parameters are those given in Tables 2 and 3.

Again, excellent agreement between DEM and experimental results is obtained using 16000 spheres (Fig. 19).

\subsubsection{Brasilian tensile strength (BTS) test on cement sample}

The BTS test was carried out for a sample of 1.487 in diameter and 0.863 in thickness. The density of the material was $1.70 \mathrm{~g} / \mathrm{cm}^{3}$. The experimental value of the maximum load in the BTS test was $847 \mathrm{lb}$ which corresponds to a value of $\left(\sigma_{t}^{f}\right)_{B T S}=420 \mathrm{Psi} \approx 2.9 \mathrm{MPa}$. Hence, $\sigma_{t}^{f}=1.6\left(\sigma_{t}^{f}\right)_{B T S}=$ 4.8 Mpa (Sect. 7.1).

The DEM parameters used are given in Tables 2 and 3.
Table 3 DEM constitutive parameters for the UCS, USC and BTS tests on cement samples

Table 4 DEM constitutive parameters for triaxial tests on cement samples

\begin{tabular}{lllllllll}
\hline LCS1 $(\mathrm{MPa})$ & LCS2 $(\mathrm{MPa})$ & LCS3 $(\mathrm{MPa})$ & YRC1 & YRC2 & YRC3 & $\delta_{n}$ & $\delta_{s}$ & $\alpha$ \\
\hline 8.5 & 9.0 & 11 & 3 & 9 & 24 & 0.20 & 0.2 & 1.0 \\
\hline
\end{tabular}

\begin{tabular}{llllllllll}
\hline $\begin{array}{l}\text { Confining } \\
\text { pressure (Psi) }\end{array}$ & $\begin{array}{l}\text { LCS1 } \\
(\mathrm{MPa})\end{array}$ & $\begin{array}{l}\text { LCS2 } \\
(\mathrm{MPa})\end{array}$ & $\begin{array}{l}\text { LCS3 } \\
(\mathrm{MPa})\end{array}$ & YRC1 & YRC2 & YRC3 & $\delta_{n}$ & $\delta_{s}$ & $\alpha$ \\
\hline 500 & 9.5 & 11 & 13 & 3 & 9 & 24 & 0.20 & 0.2 & 1.0 \\
1000 & 10 & 11 & 14 & 3 & 9 & 24 & 0.20 & 0.2 & 1.0 \\
2000 & 13 & 14 & 15 & 3 & 9 & 24 & 0.20 & 0.2 & 1.0 \\
4000 & 21 & 23 & 26 & 3 & 9 & 24 & 0.20 & 0.2 & 1.0 \\
\hline
\end{tabular}

Fig. 15 DEM and experimental results for uniaxial compressive strength (UCS) test in a cement sample. DEM results for 42000 spheres

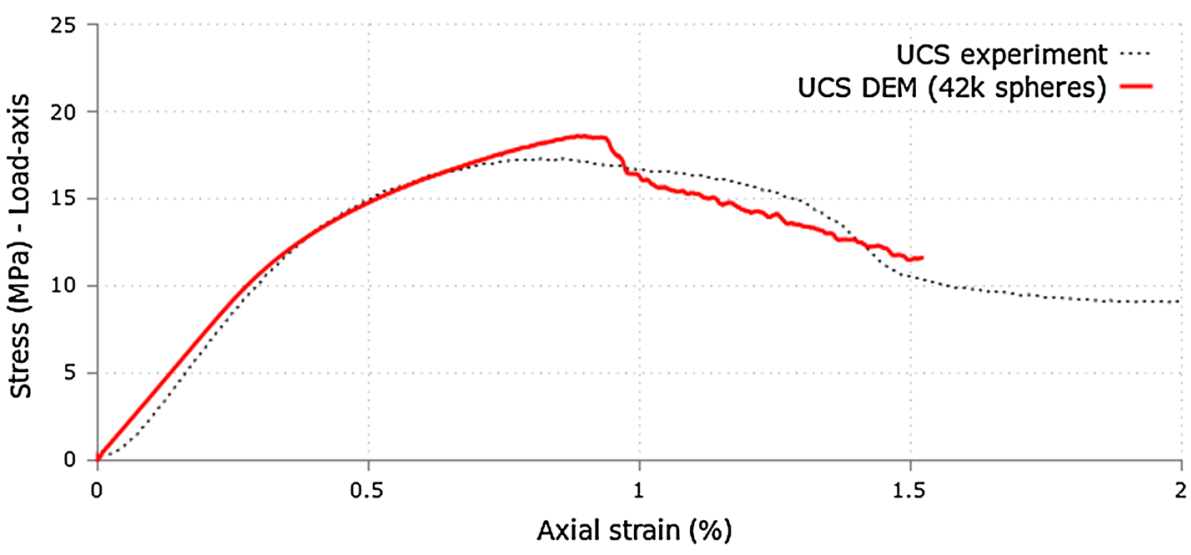


Fig. 16 Triaxial tests in cement samples. DEM-Drill and experimental results for confining pressures of a 500Psi, b 1000 Psi. DEM results for 42000 spheres. Triaxial tests in cement samples. DEM and experimental results for confining pressures of $\mathbf{c}$ 2000 Psi and d 4000 Psi. DEM results for 42000 spheres (a) Confining pressure $=500 \mathrm{psi}$

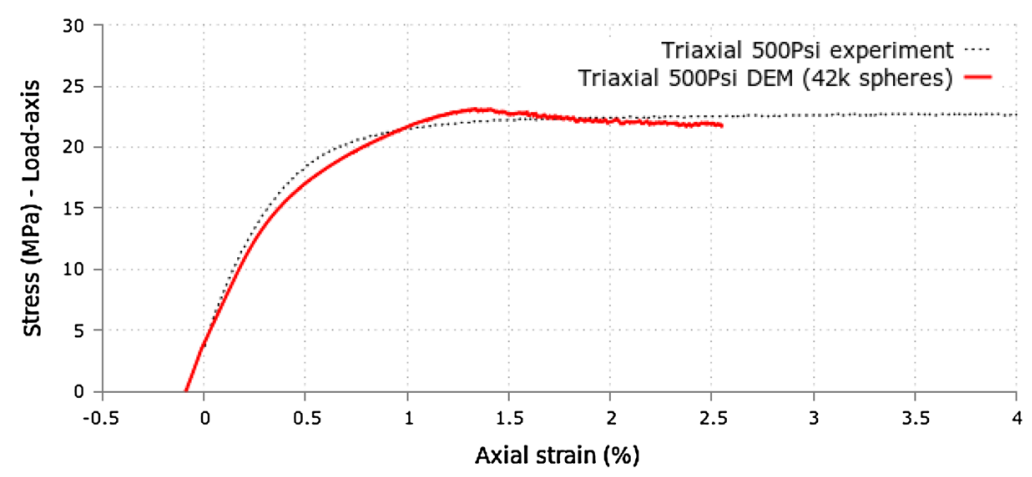

(b) Confining pressure $=1000 \mathrm{psi}$

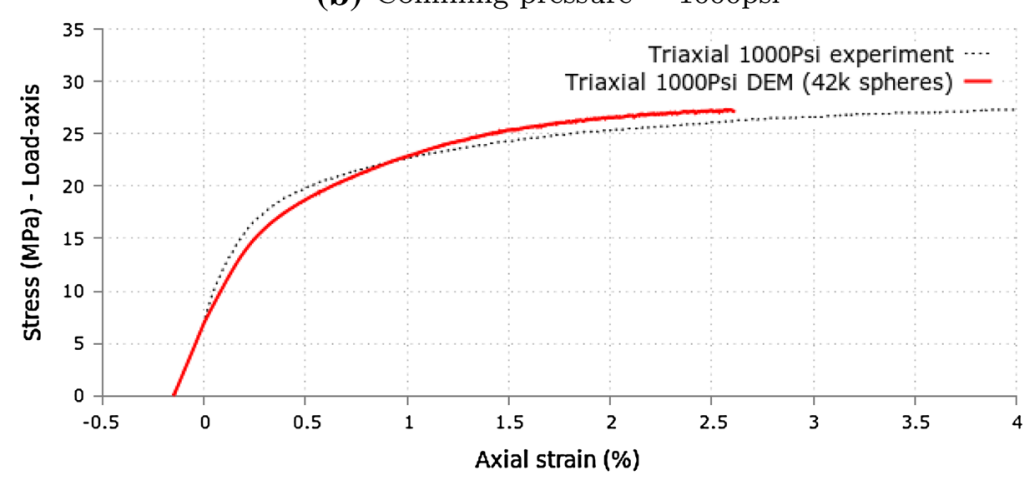

(c) Confining pressure $=2000 p s i$

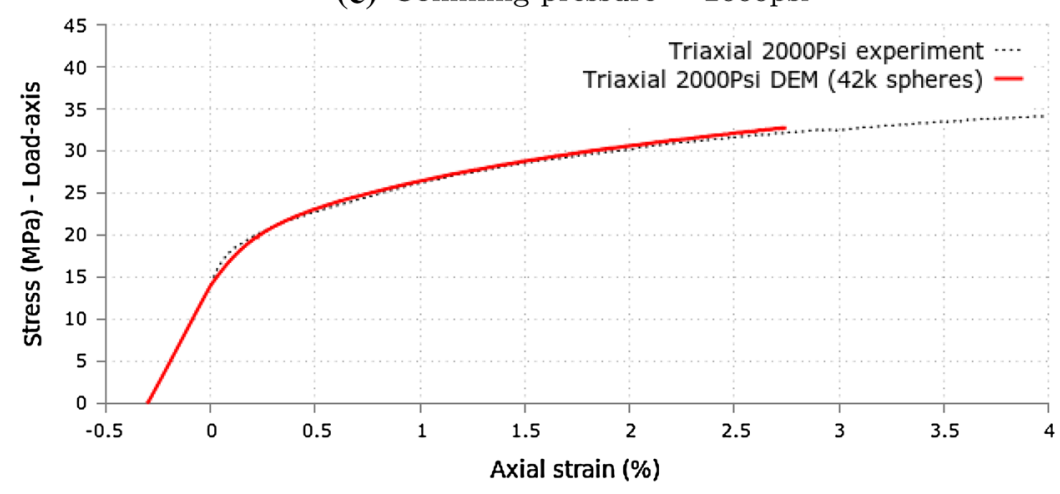

(d) Confining pressure $=4000 p s i$

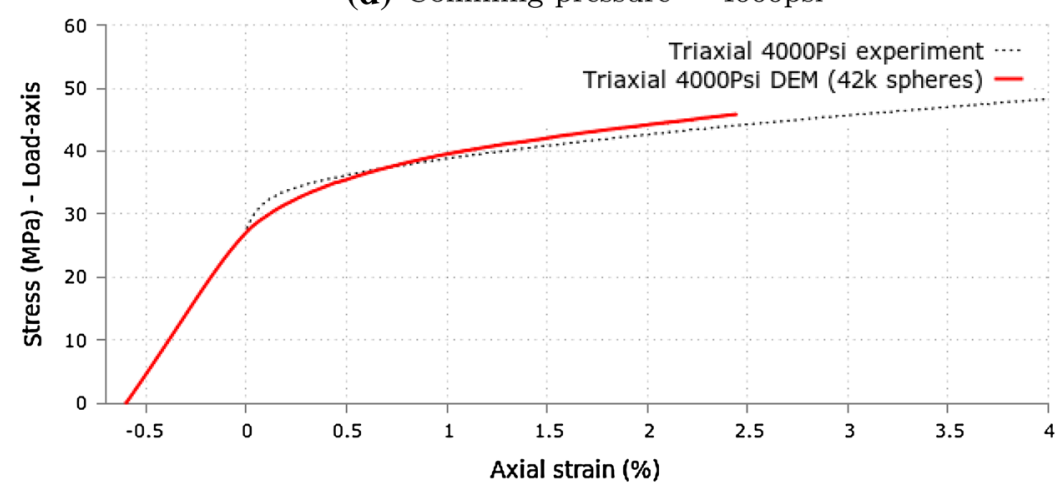




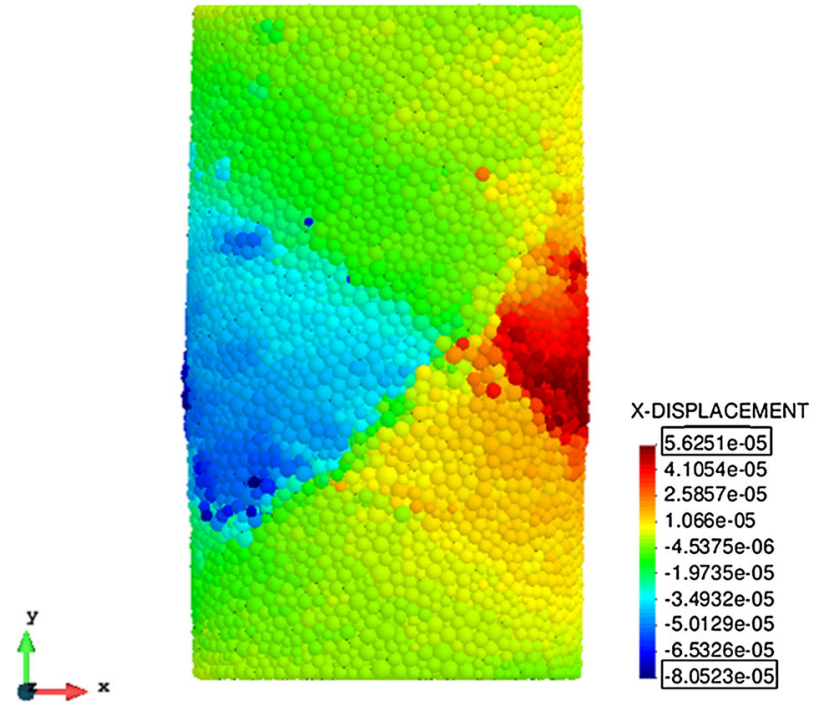

GiD

Fig. 17 UCS fracturing in cylindrical cement sample
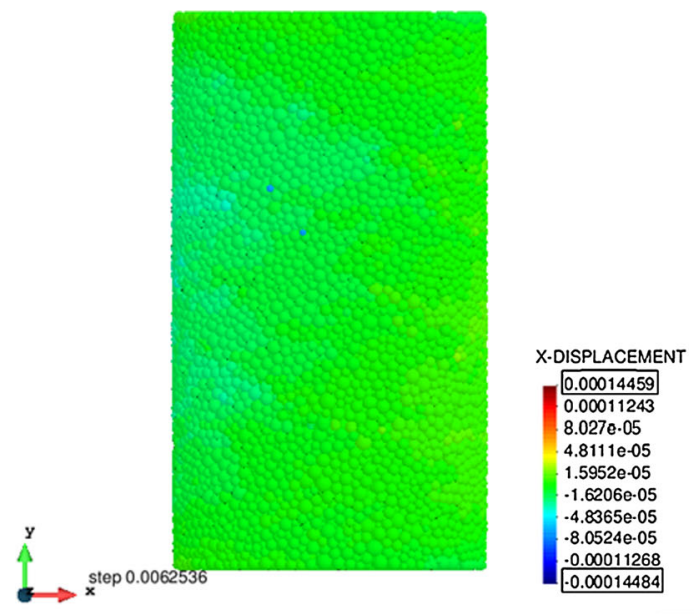

GiD

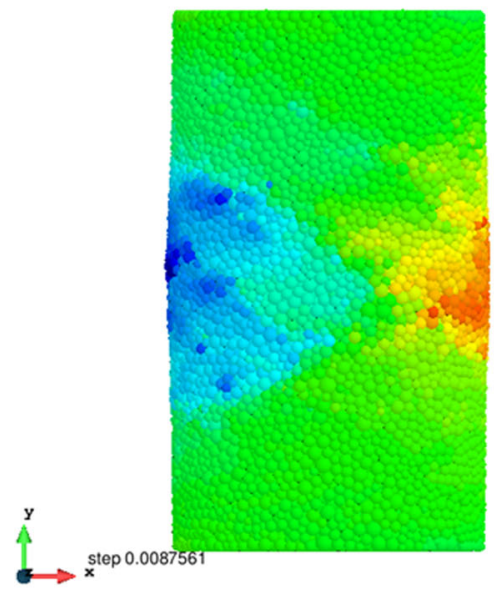

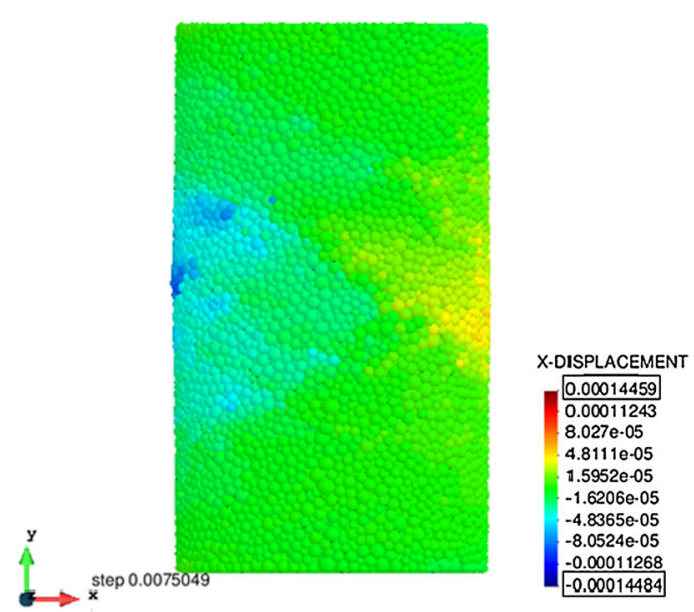

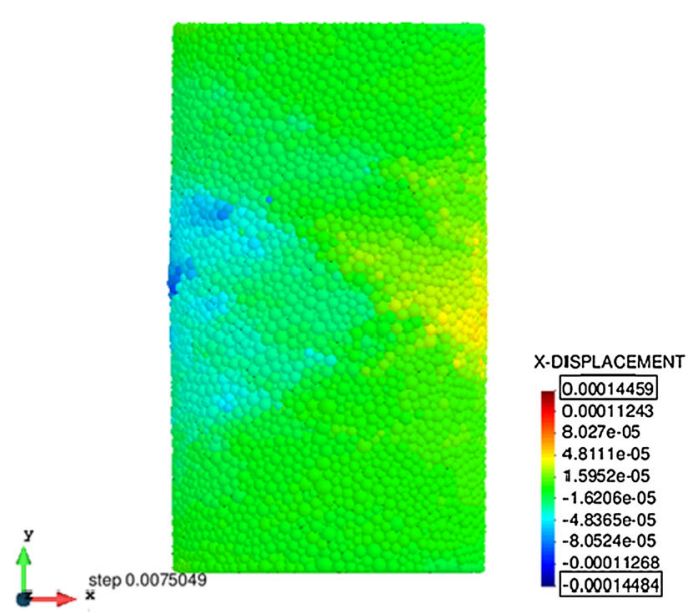

The DEM analysis was carried out using a discretization of 16000 spheres. The stress-displacement curve obtained with the DEM is displayed in Fig. 20. The maximum tensile strength computed is 3.0 MPa. The agreement with the experimental value of $2.9 \mathrm{MPa}$ is within $3.5 \%$ of relative error.

Figure 21 shows the $x$-displacement field on the sample once it has broken.

\subsection{DEM analysis of laboratory tests on concrete samples}

The experimental tests were carried out at the laboratories of the Technical University of Catalonia (UPC) in Barcelona, Spain. Details of the test are given in [37]. The concrete used in the experimental study was designed to have a characteristic compressive strength of between 32.8 and $38 \mathrm{MPa}$ at 28 days. Standard cylindrical specimens (of $150 \mathrm{~mm}$ diameter

GiD

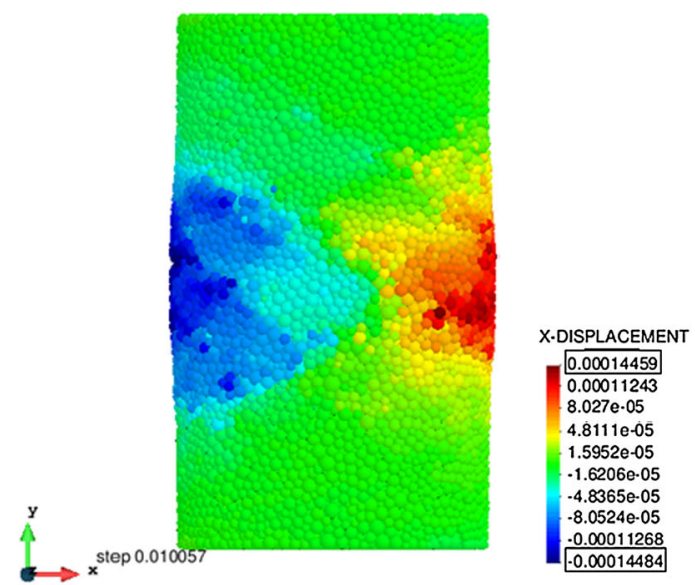

Fig. 18 Triaxial test under 500 Psi confinement in cement sample at 1.28, 1.55, 1.80, and $2.0 \%$ axial strain 
Fig. 19 DEM results for uniaxial strain compaction (USC) test on cement sample using 16000 spheres
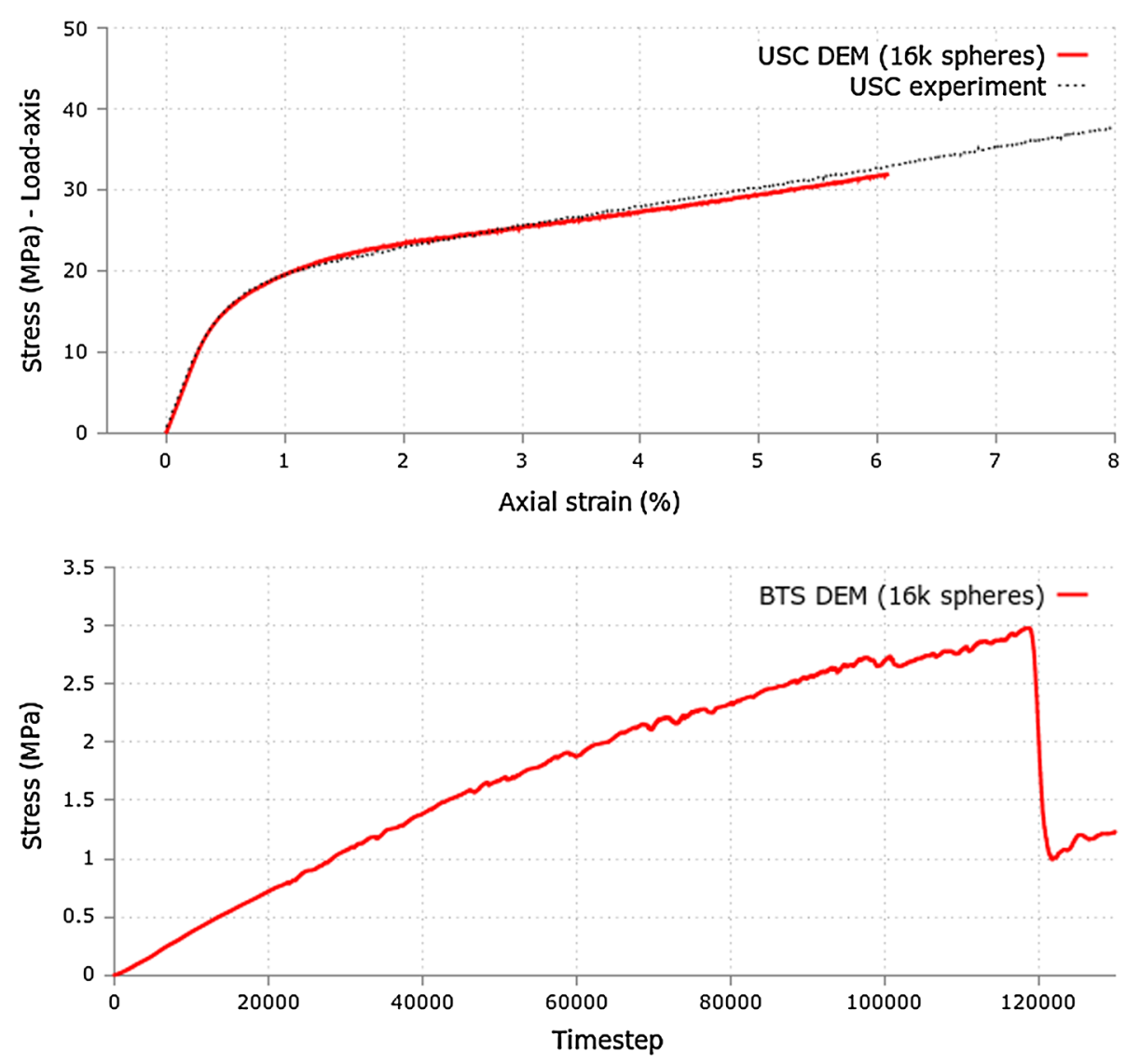

Fig. 20 DEM results for Brasilian strength (BTS) test in cement sample using 16000 spheres
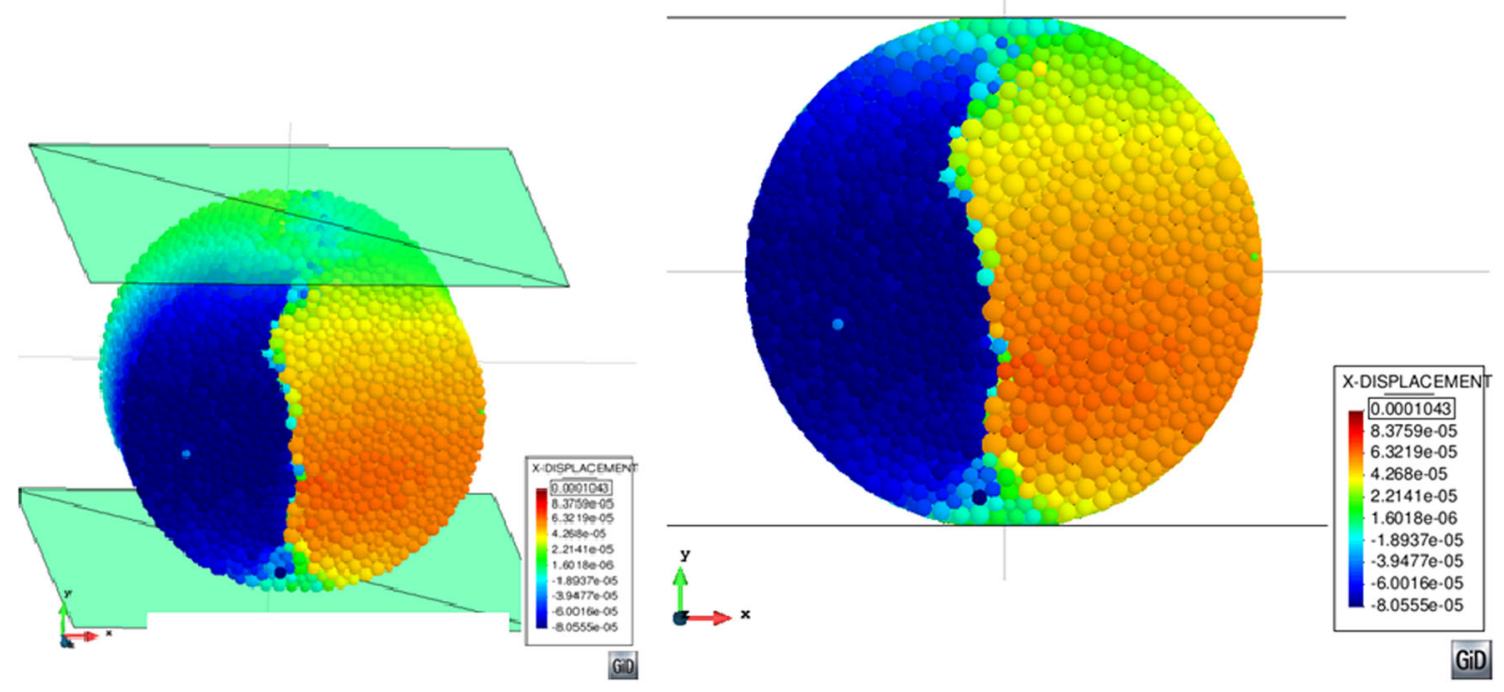

Fig. 21 DEM results for the x-displacement for BTS test in cement sample using 16000 spheres

and $300 \mathrm{~mm}$ height) were cast in metal molds and demolded after $24 \mathrm{~h}$ for storage in a fog room.

The triaxial tests were prepared with a 3-mm-thick butyl sleeve placed around the cylinder and an impermeable neoprene sleeve fitted over it. Before placing the sleeves, two pairs of strain gages were glued on the surface of the specimen at mid-height. Steel loading platens were placed at the flat ends of the specimen and the sleeves were tightened over them with metal scraps to avoid the ingress of oil.

The tests were performed using a servo-hydraulic testing machine with a compressive load capacity of 4.5 MN and a pressure capacity of $140 \mathrm{MPa}$. The axial load from the testing machine is transmitted to the specimen by a piston that passes through the top of the cell. Several levels of confining 


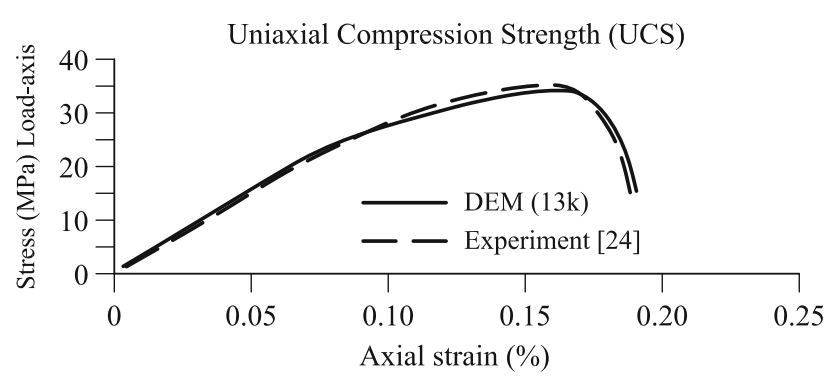

Fig. 22 Uniaxial compression strength (UCS) test in concrete sample. DEM results for 13000 spheres and experimental values

pressure ranging from 1.5 to $60 \mathrm{MPa}$ were used in order to study the brittle-ductile transition of the response. First the prescribed hydrostatic pressure was applied in the cell, and then the axial compressive load was increased at a constant displacement rate of $0.0006 \mathrm{~mm} / \mathrm{s}$.

Two specimens were tested at each confining pressure, and all tests were performed at ages of more than 50 days to minimize the effect of aging response. In addition to the triaxial tests, uniaxial compression tests were also performed.

Concrete samples were tested in dry conditions. For our computations the limit compressive normal stress was estimated as the stress level of the axial stress-deformation curve where elasto-plastic behaviour initiates in the UCS test [37] (Fig. 22) and taking into account the correction mentioned in Sect. 9. This gives $\sigma_{n_{c}}^{l}=15 \mathrm{Mpa}$.

On the other hand, the value of $\sigma_{t}^{f}$ was estimated using Eq. (43) for a value of $\left(\sigma_{n_{c}}^{f}\right)_{U C S}=37 \mathrm{MPa}$. This gives $\sigma_{t}^{f} \simeq$ $5 \mathrm{MPa}$. As for $\tau^{f}$ we have taken $\tau^{f}=0.45\left(\sigma_{n_{f}}^{f}\right)_{U C S} \simeq 16$ $\mathrm{MPa}$.

The DEM constitutive parameters for the analysis of the concrete samples are shown in Table 5. Figures 22 and 23 respectively show DEM results for the UCS and triaxial tests for confining pressures of 4.5, 9.0 and $60 \mathrm{MPa}$ using a discretization of 13000 spheres. Results for the BTS test are shown in Fig. 24. Good agreement with the experimental results [37] was found in all cases.

Figure 25 shows results of a triaxial test for the same concrete material and a confining pressure of $40 \mathrm{MPa}$. The effect of plasticity for different unloading-reloading paths is accurately predicted with the DEM using just 4000 spheres.

\subsection{UCS and BTS tests on shale rock material}

We have simulated with the DEM code a UCS test and a BTS test on a shale rock material corresponding to a Middle Brown gaseous shale in Devonian formation from Lincoln County, West Virginia. The essential material parameters for the DEM simulations were taken from [33].

The simulations were carried out in a cylindrical sample of dimensions $150 \times 300 \mathrm{~mm}$. The material properties used for the DEM analysis are listed in Table 6. The value of $\tau^{f}=25 \mathrm{MPa}$ was obtained using Eq. (44) with $\beta=0.5$ and $\left(\sigma_{n_{f}}^{f}\right)_{U C S}=50 \mathrm{MPa}$ as reported in [33].

The curve in Fig. 26 shows the axial stress-axial strain curve for the UCS test. A maximum compressive stress of $48 \mathrm{MPa}$ was obtained using a discretization of 37000 spheres. This yields a $4 \%$ error versus the experimental value of 50 MPa [33].

The curve in Fig. 27 shows the tensile stress-versus time for the BTS test obtained with the DEM using 27000 spheres. A failure tensile stress of $\sigma_{t}^{f}=5.4 \mathrm{MPa}$ was obtained. This gives a $8 \%$ error versus the experimental value of $\sigma_{t}^{f}=5$ MPa.

\section{Concluding remarks}

We have presented a local constitutive model for the DEM. The model governs the linear and non linear relationships between the normal and tangential forces and the corresponding relative displacements at the contact interfaces between discrete particles. The good behaviour of the model has been verified in its application to the analysis with the DEM of cement, concrete and shale rock samples under different strength tests. DEM results compare well with experimental data for the same tests.

The results obtained in this work show that the DEM model presented yields an accurate and reliable numerical method for linear and nonlinear analysis of geomaterials and concrete under mechanical loading.

Further validation of the DEM model presented is still needed in order to asses its convergence features for the non linear analysis of cohesive material in terms of the number and size distribution of the spheres.
Table 5 DEM constitutive parameters for tests on concrete samples

\begin{tabular}{lllllllll}
\hline$\rho(\mathrm{g} / \mathrm{cc})$ & $\mu_{1}$ & $\mu_{2}$ & $E_{0}(\mathrm{GPa})$ & $v$ & $\sigma_{t}^{f}(\mathrm{MPa})$ & $\tau^{f}(\mathrm{MPa})$ & \\
\hline 2.5 & 0.90 & 0.25 & 28 & 0.2 & 5.0 & 16 & \\
\hline $\operatorname{LCS} 1(\mathrm{MPa})$ & LCS2 $(\mathrm{MPa})$ & LCS3 $(\mathrm{MPa})$ & YRC1 & YRC2 & YRC3 & $\delta_{n}$ & $\delta_{s}$ & $\alpha$ \\
\hline 20 & 45 & 70 & 3 & 12 & 22 & 0.2 & 0.2 & 1.0 \\
\hline
\end{tabular}


Fig. 23 Triaxial tests in concrete samples. DEM results for 13000 spheres and experimental values for confinement pressures of a 4.5 MPa, b $9.0 \mathrm{MPa}$, c $60 \mathrm{MPa}$ (a) Triaxial Load - 4.5MPa Pressure

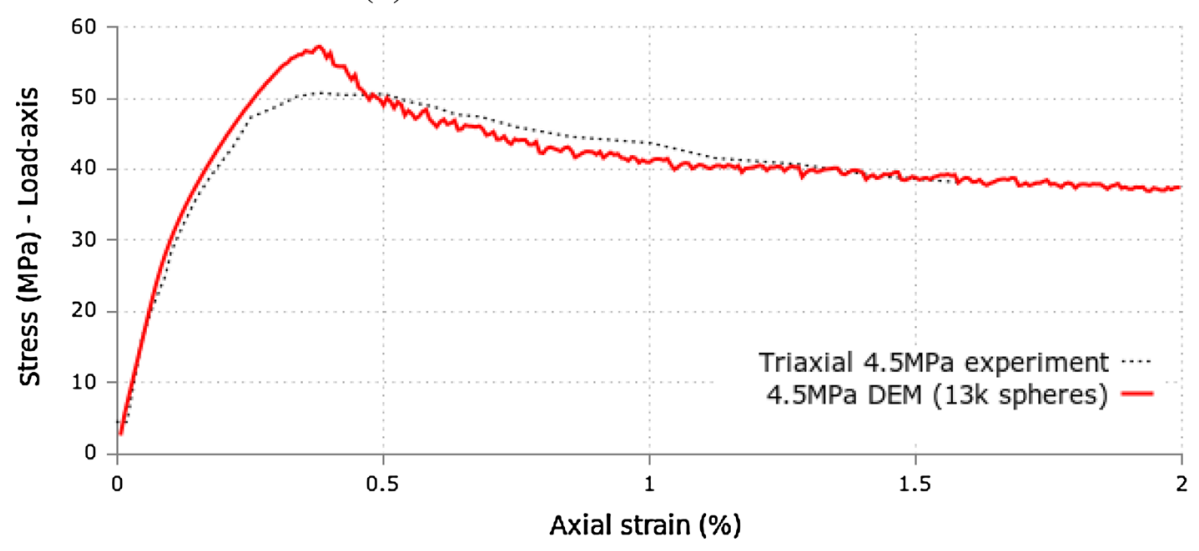

(b) Triaxial Load - 9.0MPa Pressure

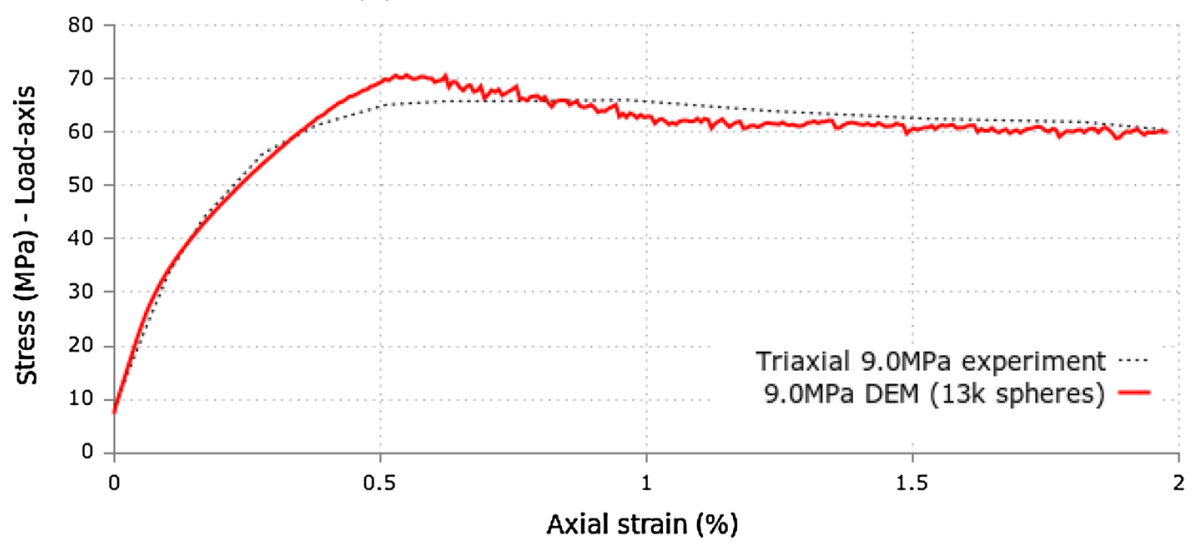

(c) Triaxial Load - 60MPa Pressure

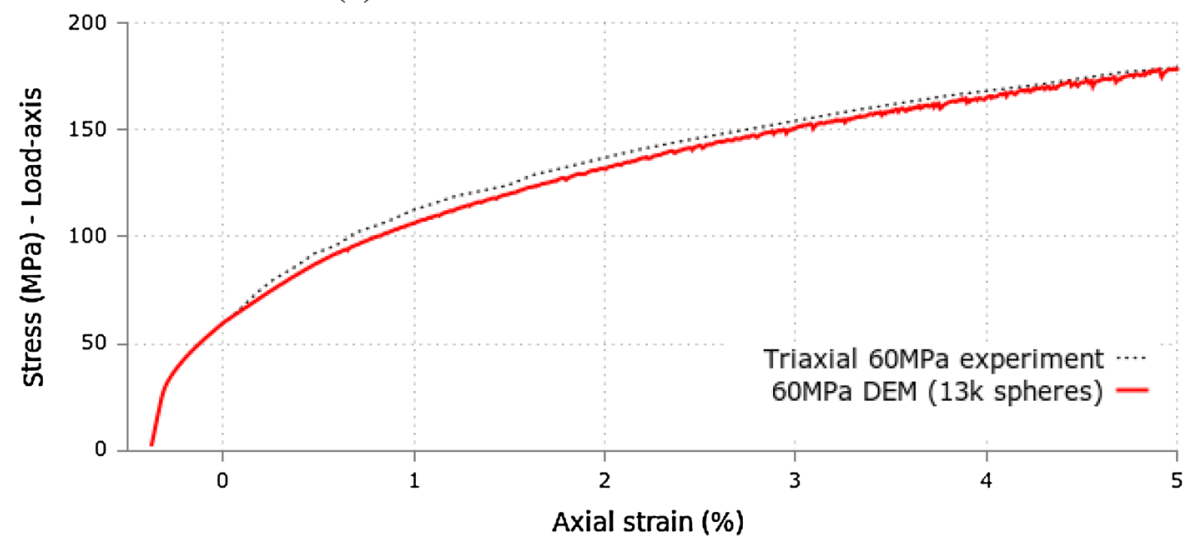


Fig. 24 BTS test in concrete sample. DEM results for 27000 spheres. The experimental limit tensile stress is $3 \mathrm{Mpa}$

Fig. 25 Triaxial test in concrete sample. DEM results for 4000 spheres and experimental values [37] for a confinement pressure of $40 \mathrm{MPa}$. Results show the effect of plasticity for different unloading-reloading paths
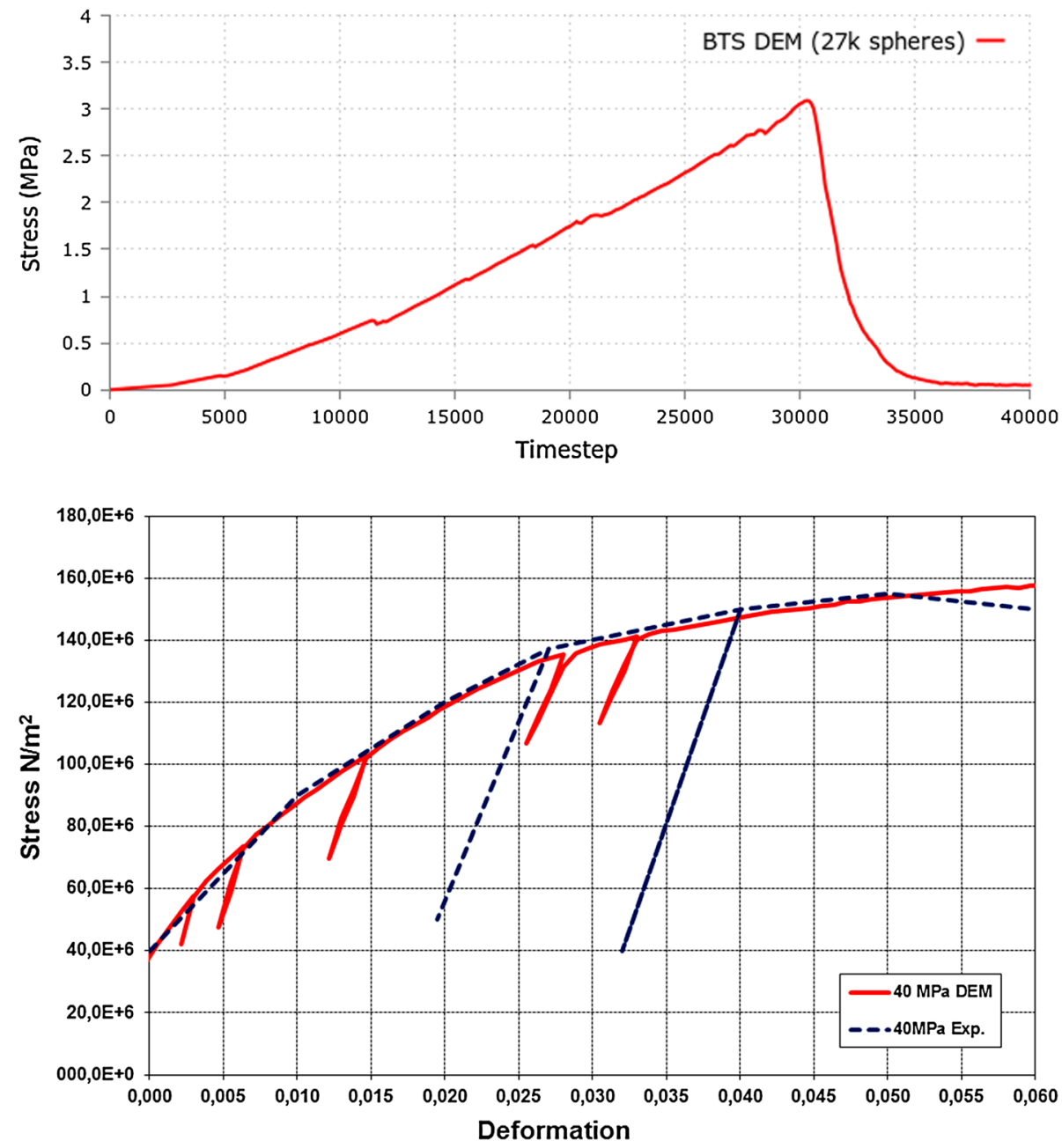

Table 6 DEM constitutive parameters for tests on shale rock samples

\begin{tabular}{lllllllll}
\hline$\rho(\mathrm{g} / \mathrm{cc})$ & $\mu_{1}$ & $\mu_{2}$ & $E_{0}(\mathrm{GPa})$ & $v$ & $\sigma_{t}^{f}(\mathrm{MPa})$ & $\tau^{f}(\mathrm{MPa})$ & \\
\hline 2.55 & 0.7 & 0.6 & 30 & 0.2 & 5.0 & 25 & \\
\hline $\operatorname{LCS} 1(\mathrm{MPa})$ & $\operatorname{LCS} 2(\mathrm{MPa})$ & $\operatorname{LCS} 3(\mathrm{MPa})$ & YRC1 & YRC2 & YRC3 & $\delta_{n}$ & $\delta_{s}$ & $\alpha$ \\
\hline 20 & 30 & 40 & 1 & 1 & 1 & 0.2 & 0.2 & 1.0
\end{tabular}

Fig. 26 Axial stress-axial strain curve for UCS test in shale rock material. DEM results using 37000 spheres

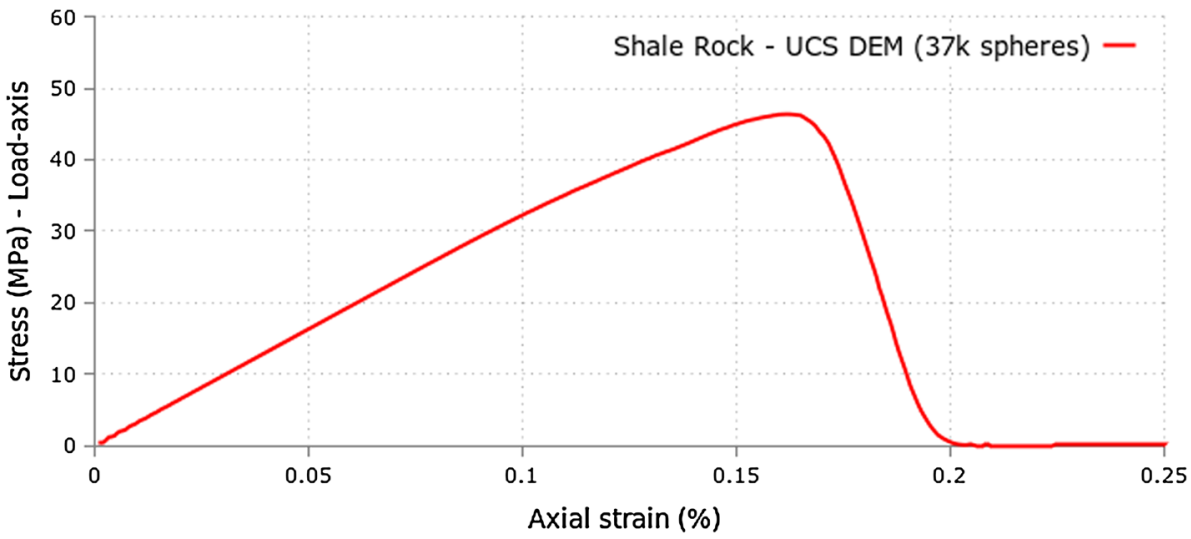


Fig. 27 Tensile stress-time curve for BTS test in shale rock material. DEM results using 27000 spheres

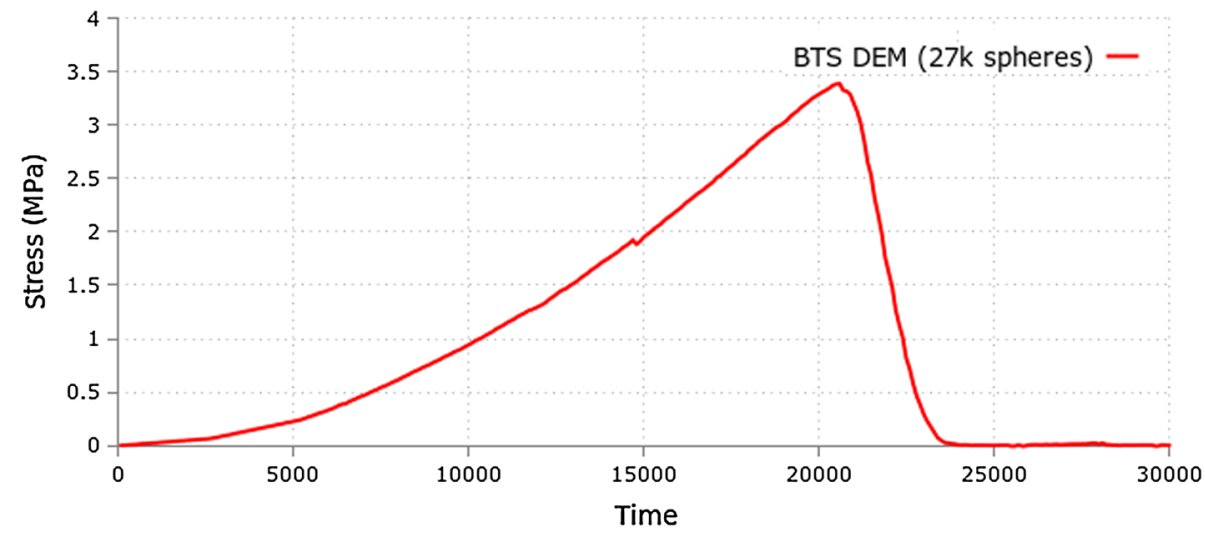

Acknowledgments This work was carried out with financial support from Weatherford, the Advanced Grant Projects SAFECON and COMDESMAT of the European Research Council and the BALAMED project (BIA2012-39172) of MINECO, Spain. The support of CIMNE for making available the codes DEMPACK (www.cimne. com/dempack), KRATOS (www.cimne.com/kratos) and GiD (www. gidhome.com) is gratefully acknowledged.

\section{Annex}

We list below the key analysis parameters involved in the DEM model presented. All the parameters, except $\alpha$ are macroscopic material parameters that should be determined from experimental tests.

$\begin{array}{ll}\rho: & \text { Density } \\ \mu_{1}: & \text { Static (Coulomb) friction coefficient } \\ \mu_{2}: & \text { Dynamic (Coulomb) friction coeffi- } \\ & \text { cient } \\ E_{0}: & \text { Young modulus at the onset of the } \\ & \text { uniaxial compression tests } \\ \nu: & \text { Poisson's ratio } \\ \sigma_{t}^{f}: & \text { Tensile failure stress } \\ \tau^{f}: & \text { Shear failure stress } \\ \text { LCS1,LCS2,LCS3: }^{f} & \text { Values of limit compressive stress } \\ & \text { defining changes on the uniaxial } \\ & \text { stress-strain relationship (Fig. 10) } \\ \text { YRC1,YRC2,YRC3 } & \text { Reduction parameters for the initial } \\ & \text { Young modulus } E_{0} \text { at the limit com- } \\ & \text { pressive stress values LCS1, LCS2 } \\ & \text { and LCS3, respectively } \\ & \text { Non dimensional damage parame- } \\ & \text { ters in the normal and shear direc- } \\ & \text { tions [Eq. (47)] } \\ & \text { Parameter that accounts for the num- } \\ \delta_{n}, \delta_{s}: & \text { ber of contacts and packaging dis- } \\ & \text { tribution of spheres in the DEM } \\ & \text { mesh. This is a model parameter } \\ & \text { that depends on the distribution of } \\ & \text { spheres in the DEM mesh. } \\ & \end{array}$

\section{References}

1. Agnolin I, Roux J-N (2008) On the elastic moduli of threedimensional assemblies of spheres: characterization and modeling of fluctuations in the particle displacement and rotation. Int J Solids Struct 45:1101-1123

2. American Society for Testing and Material Standard test method for splitting tensile strength of cylindrical concrete specimens (2004) ASTM standard C4996M-04, Philadelphia

3. Anderson JT, Gallup RF, Russell FA (2012) Shearing strength of concrete. Biblioscholar, USA, p 44

4. Avci B, Wriggers P (2012) A DEM-FEM coupling approach for the direct numerical simulation of 3D particulate flows. J Appl Mech 79(1):7

5. Balbo JT (2013) Relations between indirect tensile and flexural strengths for dry and plastic concretes. Rev IBRACONEstrut Mater 6(6):854-874. doi:10.1590/S1983-41952013000600003

6. Chang C, Hicher P-Y (2005) An elasto-plastic model for granular materials with microstructural consideration. Int J Solids Struct 42:4258-4277

7. Cheung G, O'Sullivan C (2008) Effective simulation of flexible lateral boundaries in two- and three dimensional DEM simulations. Particuology 6:483-500

8. Cleary PW (2009) Industrial particle flow modelling using DEM. Eng Comput 29:698-793

9. Cook JM, Sheppard MC, Houwen OH (1991) Effects of strain rate and confining pressure on the deformation and failure of shale. SPE Drill Eng 6(2):100-104

10. Concrete properties. The engineering tool box (2015) www. engineeringtoolbox.com/concrete-properties-d_1223.html

11. Cundall PA, Strack ODL (1979) A discrete numerical method for granular assemblies. Geotechnique 29:47-65

12. Di Renzo A, Di Maio FP (2004) Comparison of contact-force models for the simulation of collisions in DEM-based granular flow codes. Chem Eng Sci 59:525-541

13. Donzé F, Richefeu F, Magnier S (2009) Advances in discrete element method applied to soil, rock and concrete mechanics in state of the art of geotechnical engineering. Electro J Geotechnol Engg $8: 1-44$

14. Fakhimi A, Villegas T (2007) Application of dimensional analysis in calibration of a discrete element model for rock deformation and fracture. Rock Mech Rock Eng 40(2):193-211

15. Gladwell GML (1980) Contact problems in the classical theory of elasticity. Sijthoff \& Noordhoff, Alphen aan den Rijn

16. Hentz S, Daudeville L, Donzé F (2004) Identification and validation of a discrete element model for concrete. J Eng Mech 130(6):709719

17. Hsieh Y-M, Li H-H, Huang T-H, Jeng F-S (2008) Interpretations on how the macroscopic mechanical behavior of sandstone affected 
by microscopic properties revealed by bonded-particle model. Eng Geol 99(1):1-10

18. Huang H (1999) Discrete element modeling of tool-rock interaction. Ph.D. Thesis, University of Minnesota

19. Kwon O (2010) Rock mechanics testing and analyses. Cement mechanical testing. Weatherford Laboratories Report, WFT Labs RH-45733, March 2010

20. Kruyt N, Rothenburg L (2004) Kinematic and static assumptions for homogenization in micromechanics of granular materials. Mech Mater 36(12):1157-1173

21. Labra C, Oñate E (2009) High-density sphere packing for discrete element method simulations. Commun Numer Methods Eng 25(7):837-849

22. Labra C, Rojek J, Oñate E, Zárate F (2009) Advances in discrete element modelling of underground excavati ons. Acta Geotech 3(4):317-322

23. Labra C (2012) Advances in the development of the discrete element method for excavation processes. Ph.D. Thesis. Technical University of Catalonia, UPC, July 2012

24. Liao C-L, Chan T-C, Young D-H, Chang CS (1995) Stress-strain relationship for granular material based on the hypothesis of best fit. Int J Solids Struct 34(31-32):4087-4100

25. Liao C-L, Chan T-C (1997) A generalized constitutive relation for a randomly packed particle assembly. Comput Geomech 20(34):345-363

26. Lubliner J, Oller S, Oliver J, Oñate E (1989) A plastic damage model for concrete. Int J Solids Struct 25(3):299-326

27. Mindlin RD (1949) Compliance of elastic bodies in contact. J Appl Mech 16:259-268

28. Mustoe GWG (1992) A generalized formulation of the discrete element method. Eng Comput 9(2):181-190 (Special issue on Discrete Element Methods, Editor: G. Mustoe)

29. Ng T-T (2006) Input parameters of discrete element methods. J Eng Mech 132(7):723-729

30. Oller S, Botello S, Miquel J, Oñate E (1995) An anisotropic elastoplastic model based on an isotropic formulation. Eng Comput 12(3):245-262

31. Oñate E, Rojek J (2004) Combination of discrete element and finite element methods for dynamic analysis of geomechanics problems. Comput Methods Appl Mech Eng 193:3087-3128

32. Potyondy D, Cundall P (2004) A bonded-particle model for rock. Int J Rock Mech Min Sci 41(8):13291364 Rock Mechanics Results from the Underground Research Laboratory, Canada, 2004

33. Reza Safari M, Gandikota R, Mutlu U, Ji M, Glanville J, Abass H (2013) Pulsed fracturing in shale reservoirs: geomechanical aspects, ductile-brittle transition and field implications. Presented at the unconventional resources technology conference, Denver, CO, USA, 12-14 Aug 2013
34. Rojek J, Oñate E, Zarate F, Miquel J (2001) Modelling of rock, soil and granular materials using spherical elements. In: 2nd European conference on computational mechanics ECCM-2001, Cracow, 26-29 June 2001

35. Rojek J, Oñate E (2007) Multiscale analysis using a coupled discrete/finite element model. Interact Multiscale Mech 1(1):1-31

36. Rojek J, Labra C, Su O, Oñate E (2012) Comparative study of different micromechanical parameters. Int J Solids Struct 49:14971517

37. Sfer D, Carol I, Gettu R, Etse G (2002) Study of the behaviour of concrete under triaxial compression. J Eng Mech 128(2):156-163

38. Shiu W, Donzé FV, Daudeville L (2008) Compaction process in concrete during missile impact: a DEM analysis. Comput Concr 5(4):329-342

39. Sone H (2012) Mechanical properties of shale gas reservoir rocks and its relation to the in-situ stress variation observed in shale gas reservoirs. Ph.D. Thesis, Dept. of Geophysics, Standford Univ., USA, SRB vol 128, March 2012

40. Steiger RP, Leung PK (1988) Quantitative determination of the mechanical properties of shales. In: SPE-18024, 63rd annual technical conference and exhibition of society of petroleum engineers, Houston, 2-5 Oct 1988

41. Talbot AN (1906) Test of concrete: I Shear; II Bond. Engineering Experimental Station, Univ. Illinois, Bulletin No. 8, Sept 1906. www.ideals.illinois.edu/bitstream/handle/2142/4488/ engineeringexperv00000i00008? sequence $=3$

42. Tavarez FA, Plesha ME (2007) Discrete element method for modelling solid and particulate materials. Int J Numer Methods Eng 70(4):379-404

43. Tran VT, Donzé F-V, Marin P (2011) A discrete element model of concrete under high triaxial loading. Cem Concr Compos 33:936948

44. Wang H-C, Zhao W-H, Sun D-S, Guo B-B (2012) MohrCoulomb yield criterion in rock plastic mechanics. Chin J Geophys 55(6):733-741

45. Wellmann C, Lillie C, Wriggers $P$ (2008) Homogenization of granular material modeled by a three-dimensional discrete element method. Comput Geotech 35:394-405

46. Wellmann C, Lillie C, Wriggers P (2008) Comparison of the macroscopic behavior of granular materials modeled by different constitutive equations on the microscale. Finite Elem Anal Des 44:259-271

47. Williams JR, O’Connor R (1999) Discrete element simulation and the contact problem. Arch Comput Methods Eng 6(4):279-304

48. Zienkiewicz OC, Taylor RL (2005) The finite element method for solid and structural mechanics, 6th edn. Elsevier, Oxford 